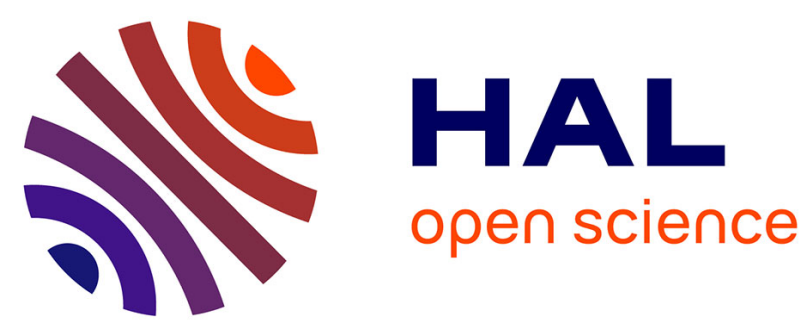

\title{
Convergence of multi-revolution composition time-splitting methods for highly oscillatory differential equations of Schrödinger type
}

Philippe Chartier, Florian Méhats, Mechthild Thalhammer, Yong Zhang

\section{To cite this version:}

Philippe Chartier, Florian Méhats, Mechthild Thalhammer, Yong Zhang. Convergence of multi-revolution composition time-splitting methods for highly oscillatory differential equations of Schrödinger type. ESAIM: Mathematical Modelling and Numerical Analysis, 2017, 51 (5), pp.1859 1882. 10.1051/m2an/2017010 . hal-01636323

\section{HAL Id: hal-01636323 \\ https://hal.science/hal-01636323}

Submitted on 16 Nov 2017

HAL is a multi-disciplinary open access archive for the deposit and dissemination of scientific research documents, whether they are published or not. The documents may come from teaching and research institutions in France or abroad, or from public or private research centers.
L'archive ouverte pluridisciplinaire HAL, est destinée au dépôt et à la diffusion de documents scientifiques de niveau recherche, publiés ou non, émanant des établissements d'enseignement et de recherche français ou étrangers, des laboratoires publics ou privés. 
ESAIM: M2AN 51 (2017) 1859-1882

DOI: $10.1051 / \mathrm{m} 2 \mathrm{an} / 2017010$
ESAIM: Mathematical Modelling and Numerical Analysis

www.esaim-m2an.org

\title{
CONVERGENCE OF MULTI-REVOLUTION COMPOSITION TIME-SPLITTING METHODS FOR HIGHLY OSCILLATORY DIFFERENTIAL EQUATIONS OF SCHRÖDINGER TYPE
}

\author{
Philippe Chartier $^{1}$, Florian Méhats ${ }^{2}$, Mechthild Thalhammer $^{3}$ \\ AND YONG ZHANG ${ }^{4}$
}

\begin{abstract}
The convergence behaviour of multi-revolution composition methods combined with timesplitting methods is analysed for highly oscillatory linear differential equations of Schrödinger type. Numerical experiments illustrate and complement the theoretical investigations.
\end{abstract}

Mathematics Subject Classification. 34K33, 34G10, 35Q41, 65M12, 65N15.

Received April 11, 2016. Revised February 11, 2017. Accepted March 7, 2017.

\section{INTRODUCTION}

\subsection{Time integration of highly oscillatory Schrödinger equations}

Relevant applications including the evolution of Bose-Einstein condensates under strongly anisotropic external potentials and the long-term propagation of waves in the presence of small potentials give reasons for the study of highly oscillatory time-dependent Schrödinger equations; as well, suitable rescalings of the cubic or quintic Schrödinger equations with small initial data have this nature. Linear Schrödinger equation of this type have been considered in [10]; the more demanding nonlinear case is treated in $[3,4,6,14,15]$.

So far, numerical simulations for highly oscillatory Schrödinger equations have been a challenge, since the efficiency of established time integration methods such as operator splitting methods is significantly affected by the necessity to choose the time increments sufficiently small, in order to resolve the rapid oscillations. The recent contribution [7] provides numerical experiments which confirm that an alternative approach based on multi-revolution composition methods (MRCMs) in combination with splitting methods is favourable. In this work, our main objective is to provide a rigorous convergence analysis for this class of time integration methods applied to highly oscillatory Schrödinger equations.

Keywords and phrases. Highly oscillatory differential equations, time-dependent Schrödinger equations, multi-revolution composition methods, operator splitting methods, local error, convergence.

1 INRIA-Rennes, IRMAR, ENS Rennes, Campus de Beaulieu, 35042 Rennes cedex, France. Philippe.Chartier@inria.fr

2 Université de Rennes 1, INRIA-Rennes, IRMAR, Campus de Beaulieu, 35042 Rennes cedex, France.

Florian.Mehats@univ-rennes1.fr

3 Leopold-Franzens Universität Innsbruck, Institut für Mathematik, Technikerstraße 13/VII, 6020 Innsbruck, Austria.

Mechthild.Thalhammer@uibk. ac . at

4 Wolfgang Pauli Institut c/o Universität Wien, Fakultät für Mathematik, Oskar-Morgenstern-Platz 1, 1090 Wien, Austria.

Yong.Zhang@univie.ac.at 


\subsection{Evolutionary Schrödinger equation and reformulation}

For the purpose of a compact formulation, we consider the evolutionary Schrödinger equation

$$
\frac{\mathrm{d}}{\mathrm{d} \tau} w(\tau)=\frac{1}{\varepsilon} A w(\tau)+B w(\tau), \quad \tau \in(0, T], \quad 0<\varepsilon \ll 1 .
$$

Our basic assumptions are that the unbounded linear operator $A: D(A) \subset X \rightarrow X$ generates a strongly continuous group of isometries on the underlying Banach space $\left(X,\|\cdot\|_{X}\right)$ and that the associated propagator $\mathcal{E}_{A}(\cdot)$ is periodic in time with period $T_{0}>0$; that is, the relation $\mathcal{E}_{A}\left(T_{0}\right)=I: X \rightarrow X$ holds. In order to reduce the intricacy of our stability and error analysis, we require $B: X \rightarrow X$ to be a bounded linear operator. Moreover, we suppose that $\frac{1}{\varepsilon} A+B: D(A) \rightarrow X$ generates a group of isometries $\left(\mathcal{E}_{\frac{1}{\varepsilon} A+B}(t)\right)_{t \in \mathbb{R}}$.

For theoretical purposes, it is useful to employ the time scaling $\tau=\varepsilon t$, which leads to a long-term problem for $u:\left[0, \frac{1}{\varepsilon} T\right] \rightarrow X: t \mapsto u(t)=w(\varepsilon t)$

$$
\frac{\mathrm{d}}{\mathrm{d} t} u(t)=A u(t)+\varepsilon B u(t), \quad t \in\left(0, \frac{1}{\varepsilon} T\right], \quad 0<\varepsilon \ll 1 ;
$$

this reformulation will be the starting point for our considerations.

The scope of applications in particular includes time-dependent Schrödinger equations with mono-frequent linear main part. Throughout, we focus on a model equation

$$
\mathrm{i} \partial_{t} \psi(x, t)=-\Delta \psi(x, t)+\varepsilon V(x) \psi(x, t), \quad(x, t) \in \Omega \times\left(0, \frac{1}{\varepsilon} T\right], \quad 0<\varepsilon \ll 1,
$$

which comprises the Laplace operator defined on a cartesian product of bounded intervals $\Omega \subset \mathbb{R}^{d}$; a natural choice for the underlying function space is the Lebesgue-space $L^{2}(\Omega, \mathbb{C})$. We point out that the real-valued potential $V: \mathbb{R}^{d} \rightarrow \mathbb{R}$ and the final time $T>0$ are independent of the decisive small parameter $0<\varepsilon \ll 1$.

\subsection{Multi-revolution composition methods}

Multi-revolution composition methods for highly oscillatory (ordinary) differential equations were introduced and studied in [7], see also [9,21] and references given therein. The basic idea is to approximate the value of the evolution operator associated with (1.1) at an integer multiple of the period by a composition of the form

$$
\mathcal{C}_{A+\varepsilon B}\left(N_{0} T_{0}\right)=\prod_{j=1}^{r}\left(\mathcal{E}_{A-\beta_{j} \varepsilon N_{0} B}\left(-T_{0}\right) \mathcal{E}_{A+\alpha_{j} \varepsilon N_{0} B}\left(T_{0}\right)\right) \approx \mathcal{E}_{A+\varepsilon B}\left(N_{0} T_{0}\right) ;
$$

the quality of the approximation is determined by the choice of the real coefficients $\left(\alpha_{j}, \beta_{j}\right)_{j=1}^{r}$ and by the size of $H=\varepsilon N_{0}$ (see below). Without loss of generality, we may assume that the final time fulfills the relation $\frac{1}{\varepsilon} T=N N_{0} T_{0}$ with integer $N_{0}, N>0$, see Figure 1; otherwise, an additional short time integration based on a standard splitting approach is used. With regard to utility of the method, we always employ the assumption $N_{0} \gg r$ (see computational cost).

As illustration, we include multi-revolution composition methods constructed in [7]. The simplest method involving a single factor

$$
\begin{aligned}
& P=1: \quad r=1, \quad \alpha_{1}=1, \quad \beta_{1}=0, \quad \mathcal{C}_{A+\varepsilon B}\left(N_{0} T_{0}\right)=\mathcal{E}_{A+\varepsilon N_{0} B}\left(T_{0}\right), \\
& \hline T_{0} 2 T_{0} \quad \ldots \quad N_{0} T_{0} \\
& .
\end{aligned}
$$

Figure 1. Subdivision of the time interval $\left[0, \frac{1}{\varepsilon} T\right]$ used in the application of MRCMs. The quality of the approximation is determined by the increment $H=\varepsilon N_{0}$. 
leads to a first-order approximation with respect to the increment $H=\varepsilon N_{0}$, that is, the relation

$$
\mathcal{C}_{A+\varepsilon B}\left(N_{0} T_{0}\right)-\mathcal{E}_{A+\varepsilon B}\left(N_{0} T_{0}\right)=\mathcal{O}\left(H^{P+1}\right)
$$

is valid with $P=1$ (see below). Suitable choices of the coefficients $\left(\alpha_{j}, \beta_{j}\right)_{j=1}^{r}$ in dependence of $N_{0}$ permit to increase the approximation rate. For instance, a second-order method is given by

$$
\begin{aligned}
P=2: \quad r & =1, \quad \alpha_{1}=\frac{1}{2}\left(1+\frac{1}{N_{0}}\right), \quad \beta_{1}=\frac{1}{2}\left(1-\frac{1}{N_{0}}\right), \\
\mathcal{C}_{A+\varepsilon B}\left(N_{0} T_{0}\right)=\mathcal{E}_{A-\varepsilon \frac{N_{0}-1}{2} B}\left(-T_{0}\right) \mathcal{E}_{A+\varepsilon \frac{N_{0}+1}{2} B}\left(T_{0}\right) & \approx \mathcal{E}_{A+\varepsilon B}\left(N_{0} T_{0}\right) .
\end{aligned}
$$

A fourth-order method results from solving the order conditions for $r=3$, (see [7], Tab. 1); under the additional constraint $\alpha_{3}=\alpha_{1}$ and $\beta_{3}=\beta_{1}$ this yields

$$
\begin{gathered}
2 \alpha_{1}+\alpha_{2}+2 \beta_{1}+\beta_{2}=1, \\
2 \alpha_{1}^{2}+\alpha_{2}^{2}-2 \beta_{1}^{2}-\beta_{2}^{2}=\frac{1}{N_{0}}, \\
2 \alpha_{1}^{3}+\alpha_{2}^{3}+2 \beta_{1}^{3}+\beta_{2}^{3}=\frac{1}{N_{0}^{2}}, \\
\left(\alpha_{1}^{2}-\beta_{1}^{2}\right) \beta_{1}+\left(\alpha_{2}^{2}-\beta_{2}^{2}\right)\left(\alpha_{1}+\beta_{1}+\beta_{2}\right)+\left(\alpha_{1}^{2}-\beta_{1}^{2}\right)\left(\alpha_{1}+\alpha_{2}+2 \beta_{1}+\beta_{2}\right)=\frac{N_{0}-1}{2 N_{0}^{2}}, \\
2 \alpha_{1}^{4}+\alpha_{2}^{4}-2 \beta_{1}^{4}-\beta_{2}^{4}=\frac{1}{N_{0}^{3}}, \\
\left(\alpha_{1}^{3}+\beta_{1}^{3}\right) \beta_{1}+\left(\alpha_{2}^{3}+\beta_{2}^{3}\right)\left(\alpha_{1}+\beta_{1}+\beta_{2}\right)+\left(\alpha_{1}^{3}+\beta_{1}^{3}\right)\left(\alpha_{1}+\alpha_{2}+2 \beta_{1}+\beta_{2}\right)=\frac{N_{0}-1}{2 N_{0}^{3}}, \\
\left(\alpha_{1}^{2}-\beta_{1}^{2}\right) \beta_{1}^{2}+\left(\alpha_{2}^{2}-\beta_{2}^{2}\right)\left(\alpha_{1}+\beta_{1}+\beta_{2}\right)^{2}+\left(\alpha_{1}^{2}-\beta_{1}^{2}\right)\left(\alpha_{1}+\alpha_{2}+2 \beta_{1}+\beta_{2}\right)^{2}=\frac{\left(N_{0}-1\right)\left(2 N_{0}-1\right)}{6 N_{0}^{3}},
\end{gathered}
$$

with real solution given by

$$
\begin{gathered}
P=4: \quad r=3, \quad \alpha_{1}=\alpha_{3}=\frac{1}{N_{0}}\left(\frac{1}{12} c+\frac{1}{3} \frac{N_{0}^{2}}{c}+\frac{1}{3} N_{0}+\frac{1}{2}\right), \quad \alpha_{2}=-\frac{1}{6} \frac{c^{2}+\left(N_{0}-3\right) c+4 N_{0}^{2}}{c N_{0}}, \\
\beta_{1}=\beta_{3}=\frac{1}{N_{0}}\left(\frac{1}{12} c+\frac{1}{3} \frac{N_{0}^{2}}{c}+\frac{1}{3} N_{0}-\frac{1}{2}\right), \quad \beta_{2}=-\frac{1}{6} \frac{c^{2}+\left(N_{0}+3\right) c+4 N_{0}^{2}}{c N_{0}} \\
c=\sqrt[3]{10 N_{0}^{3}-18 N_{0}+6 \sqrt{N_{0}^{6}-10 N_{0}^{4}+9 N_{0}^{2}}} .
\end{gathered}
$$

\subsection{Realisation by time-splitting methods}

In our context of (low-dimensional) time-dependent Schrödinger equations, the computation of $\mathcal{E}_{A+\gamma B}\left(T_{0}\right)$ with $\gamma=\alpha_{j} \varepsilon N_{0}$ or $\mathcal{E}_{A+\gamma B}\left(-T_{0}\right)$ with $\gamma=-\beta_{j} \varepsilon N_{0}$, respectively, arising in (1.2), relies on the application of time-splitting pseudo-spectral methods, known to be favourable for this class of problems, see $[2,5,12,13,17,19]$ and references therein. As illustrated in Figure 2, an interval of length $T_{0}$ is divided into $K$ subintervals of equal length; the corresponding time stepsize is denoted by $h=\frac{1}{K} T_{0}$. The value of $\mathcal{E}_{A+\gamma B}\left(T_{0}\right)$, e.g., is replaced by a composition of the form

$$
\mathcal{S}_{A+\gamma B}^{K}\left(T_{0}\right)=\left(\prod_{k=1}^{s} \mathcal{E}_{\gamma B}\left(b_{k} h\right) \mathcal{E}_{A}\left(a_{k} h\right)\right)^{K} \approx \mathcal{E}_{A+\gamma B}\left(T_{0}\right)
$$




\begin{tabular}{lllll}
\hline 0 & $h$ & $2 h$ & $\ldots$ & $K h=T_{0}$
\end{tabular}

FiguRE 2. Subdivision used in the application of splitting methods, e.g. over the interval $\left[0, T_{0}\right]$.

The quality of the approximation is determined by the increment $h=\frac{T_{0}}{K}$.

For instance, the first-order Lie-Trotter splitting method

$$
p=1: \quad \mathcal{S}_{A+\gamma B}^{K}\left(T_{0}\right)=\left(\mathcal{E}_{\gamma B}(h) \mathcal{E}_{A}(h)\right)^{K} \approx \mathcal{E}_{A+\gamma B}\left(T_{0}\right)
$$

and the widely used second-order Strang splitting method

$$
p=2: \quad \mathcal{S}_{A+\gamma B}^{K}\left(T_{0}\right)=\left(\mathcal{E}_{\gamma B}\left(\frac{h}{2}\right) \mathcal{E}_{A}(h) \mathcal{E}_{\gamma B}\left(\frac{h}{2}\right)\right)^{K} \approx \mathcal{E}_{A+\gamma B}\left(T_{0}\right)
$$

satisfy the relation

$$
\mathcal{S}_{A+\gamma B}^{K}\left(T_{0}\right)-\mathcal{E}_{A+\gamma B}\left(T_{0}\right)=\mathcal{O}\left(h^{p}\right)
$$

with $p=1$ and $p=2$, respectively; for our purposes it is essential that additionally the factor $\gamma$ can be extracted. Again, the approximation rate can be raised by suitably adapting the real coefficients $\left(a_{k}, b_{k}\right)_{k=1}^{s}$, see for example $[5,22]$.

\subsection{Convergence analysis}

The main original contribution of this work is the derivation of a convergence result for multi-revolution composition time-splitting methods applied to time-dependent highly oscillatory Schrödinger equations that can be cast into the form (1.1). In short, we prove that the global error satisfies

$$
\text { global error }=\mathcal{O}\left(H^{P}+H h^{p}\right)
$$

and that an improved error estimate holds when the employed splitting method is symmetric. The derivation of this result relies on suitable estimates for the discretisation errors caused by multi-revolution composition methods and time-splitting methods.

(i) Discretisation error caused by multi-revolution composition methods. In the context of evolutionary Schrödinger equations (1.1), the presence of the unbounded operator $A$ requires to adapt the strategies for deducing local error estimates for multi-revolution composition methods. Contrary to [7], where infinite Taylor series expansions of $\mathcal{E}_{A+\gamma B}\left(T_{0}\right)$ have been used, it is essential to employ a stepwise expansion of the evolution operator associated with (1.1) by means of the variation-of-constants formula and to specify the remainder terms. We exemplify the approach for the simplest case (1.3a), where a repeated application of the variation-of-constants formula

$$
\begin{aligned}
\mathcal{E}_{A+\varepsilon B}\left(N_{0} T_{0}\right)= & \mathcal{E}_{A}\left(N_{0} T_{0}\right)+\varepsilon \int_{0}^{N_{0} T_{0}} \mathcal{E}_{A}\left(N_{0} T_{0}-\tau\right) B \mathcal{E}_{A}(\tau) \mathrm{d} \tau \\
& +\varepsilon^{2} \int_{0}^{N_{0} T_{0}} \int_{0}^{\tau} \mathcal{E}_{A}\left(N_{0} T_{0}-\tau\right) B \mathcal{E}_{A}(\tau-\sigma) B \mathcal{E}_{A+\varepsilon B}(\sigma) \mathrm{d} \sigma \mathrm{d} \tau
\end{aligned}
$$

together with the fundamental periodicity requirement $\mathcal{E}_{A}\left(T_{0}\right)=I$ leads to an expansion of the form

$$
\mathcal{E}_{A+\varepsilon B}\left(N_{0} T_{0}\right)=I+\varepsilon \int_{0}^{N_{0} T_{0}} f(\tau) \mathrm{d} \tau+\mathcal{O}\left(H^{2}\right)
$$


involving a $T_{0}$-periodic integrand; a decomposition of the interval $\left[0, N_{0} T_{0}\right]$ and a suitable integral transformation permit a reduction to the primary interval $\left[0, T_{0}\right]$

$$
\mathcal{E}_{A+\varepsilon B}\left(N_{0} T_{0}\right)=I+\varepsilon N_{0} \int_{0}^{T_{0}} f(\tau) \mathrm{d} \tau+\mathcal{O}\left(H^{2}\right)
$$

which suggests to consider the composition method (1.3a) and proves (1.3b) with $P=1$, that is

$$
\mathcal{E}_{A+\varepsilon B}\left(N_{0} T_{0}\right)=\mathcal{E}_{A+\varepsilon N_{0} B}\left(T_{0}\right)+\mathcal{O}\left(H^{2}\right) .
$$

(ii) Discretisation error caused by splitting methods. In order to deduce error estimates for time-splitting methods that capture the dependencies on the decisive quantities, we adapt the approach developed in our recent work [8]. Compared to other contributions that study the error behaviour of high-order splitting method for Schrödinger equations, see [19] and references therein, to justify the numerically observed superconvergent behaviour, it is essential to distinguish between non-symmetric and symmetric splitting methods.

\subsection{Computational cost}

As indicated before, our main result implies that the application of a $P$ th-order multi-revolution composition method defined by coefficients $\left(\alpha_{j}, \beta_{j}\right)_{j=1}^{r}$ in combination with a $p$ th-order splitting method defined by coefficients $\left(a_{k}, b_{k}\right)_{k=1}^{s}$ leads to the relation

$$
\operatorname{MRCM}(r, P, H), \quad \text { splitting }(s, p, h): \quad \text { global error }=\mathcal{O}\left(H^{P}+H h^{p}\right)
$$

at time $\frac{1}{\varepsilon} T=N N_{0} T_{0}$, where $H=\varepsilon N_{0}$ and $h=\frac{T_{0}}{K}$, and in total requires $N 2 r s K$ evaluations of $\mathcal{E}_{A}(\cdot)$ and of $\mathcal{E}_{B}(\cdot)$, respectively, that is, the cost is

$$
\operatorname{MRCM}(r, P, H), \quad \text { splitting }(s, p, h): \quad \operatorname{cost}=N 2 r s K=\frac{2 r}{N_{0}} s \frac{T}{\varepsilon h} .
$$

Contrary, the sole application of this splitting method for the time integration leads to the global error

$$
\text { splitting }(s, p, h): \quad \text { error }=\mathcal{O}\left(\frac{1}{\varepsilon} h^{p}\right)
$$

at a total cost of $N N_{0} s K$ evaluations of $\mathcal{E}_{A}(\cdot)$ and of $\mathcal{E}_{B}(\cdot)$, respectively, such that

$$
\text { splitting }(s, p, h): \quad \operatorname{cost}=N N_{0} s K=s \frac{T}{\varepsilon h} .
$$

Likewise, numerical experiments for the time-dependent cubic Schrödinger equation, presented in [7], confirm that second- and fourth-order multi-revolution composition methods combined with the Strang time-splitting Fourier pseudo-spectral method are beneficial. In particular, for smaller values of the decisive parameter $0<$ $\varepsilon \ll 1$, reflected in the magnitude of the final time $\frac{1}{\varepsilon} T \gg 1$, the resulting discretisation methods are superior in efficiency compared to a sole application of the Strang time-splitting Fourier pseudo-spectral method for the long-term integration; indeed, to compute the value of the fully discrete solution at the final time, the sole application of a $s$-stage splitting method with time stepsize $h=\frac{1}{K} T_{0}$ requires in total $N N_{0} s K$ spectral transforms, whereas the realisation of a $r$-stage multi-revolution composition method based on this splitting method requires $N 2 r s K$ spectral transforms. 


\subsection{Numerical experiments}

In order to confirm and complement our theoretical investigations, we present numerical experiments for different multi-revolution composition time-splitting Fourier pseudo-spectral methods, applied to linear and nonlinear test equations. The obtained results in particular confirm the improved error behaviour of symmetric splitting methods.

\subsection{Extensions}

As a rigorous convergence analysis of high-order time discretisation methods for nonlinear Schrödinger equations would overburden the present work, we focus on a detailed treatment of the linear model equation comprising the Laplace operator and a bounded potential. The considerations can be extended to Schrödinger equations defined by a self-adjoint operator, employing the associated countable complete orthonormal system of eigenfunctions, see for instance $[13,16,20]$. The restriction to the linear case significantly reduces the complexity in the derivation of stability results and error expansions. With regard to the contributions $[13,17,19]$, we expect that qualitatively the same global error estimate holds for the practically relevant case involving an unbounded nonlinear operator, for instance for the time-dependent cubic Schrödinger equation with nonlinearity defined by $B: D(B) \rightarrow L^{2}(\Omega, \mathbb{C}): u \mapsto|u|^{2} u$, under stronger regularity requirements on the exact solution.

\subsection{Notation and basic assumptions}

We denote by $\mathbb{N}=\{n \in \mathbb{Z}: n \geq 0\}$ the set of non-negative integer numbers. The composition of operators is defined downward

$$
\prod_{\ell=m}^{n} Q_{\ell}=\left\{\begin{array}{ll}
Q_{n} \ldots Q_{m}, & m \leq n, \\
I, & m>n,
\end{array} \quad m, n \in \mathbb{N}\right.
$$

We employ standard notation and results for Lebesgue and Sobolev spaces, see [1]. In particular, the Lebesgue space $L^{2}(\Omega)=L^{2}(\Omega, \mathbb{C})$ comprising all square-integrable complex-valued functions defined on a domain $\Omega \subseteq \mathbb{R}^{d}$ is endowed with inner product and associated norm given by

$$
(f \mid g)_{L^{2}}=\int_{\Omega} f(x) \overline{g(x)} \mathrm{d} x, \quad\|f\|_{L^{2}}=\sqrt{(f \mid f)_{L^{2}}}, \quad f, g \in L^{2}(\Omega) ;
$$

we note that we apply complex conjugation in the second argument. Henceforth, we focus on the case where multi-revolution composition time-splitting methods constitute efficient full discretisation methods for timedependent Schrödinger equations, that is, we tacitely assume that the parameter $0<\varepsilon \ll 1$ is relatively small such that the final time $\frac{1}{\varepsilon} T \gg 1$ is relatively large. In order to be consistent with the definition of splitting methods given in [19], it is natural to employ a formulation of multi-revolution composition methods which differs from [7]. We employ the reasonable assumptions that the considered increments $H=\varepsilon N_{0}$, related to a multi-revolution composition method, and the time stepsizes $h=\frac{1}{K} T_{0}$, related to the application of a splitting method on the interval $\left[0, T_{0}\right]$, satisfy $0<H<1$ as well as $0<h<1$ so that $H^{m+1}<H^{m}$ as well as $h^{m+1}<h^{m}$ holds for $m \in \mathbb{N}$. We may suppose that the final time of integration coincides with a multiple of the period $T_{0}$; otherwise, an additional short-time integration involving few time steps is performed, and the statement of the convergence result remains valid.

\section{Highly oscillatory SchröDinger EQUATIONS AND THEIR DISCRETISATION}

In this section, we state the general hypotheses on the considered class of highly oscillatory evolutionary Schrödinger equations; furthermore, we justify these requirements for the model equation involving the Laplacian and indicate the extension to related situations. For details on the employed functional analytic framework, we refer to $[11,18,20]$. Finally, we introduce the general format of multi-revolution composition time-splitting methods. 


\subsection{Analytical framework}

\subsubsection{Evolutionary Schrödinger equation}

Let $\left(X,\|\cdot\|_{X}\right)$ denote the underlying Banach space. Henceforth, we consider the initial value problem

$$
\left\{\begin{array}{l}
u^{\prime}(t)=A u(t)+\varepsilon B u(t), \quad t \in\left(0, \frac{1}{\varepsilon} T\right], \quad 0<\varepsilon \ll 1, \\
u(0) \text { given, }
\end{array}\right.
$$

involving an unbounded linear operator $A: D(A) \subset X \rightarrow X$ and a bounded linear operator $B: X \rightarrow X$. In order to indicate the dependence of the solution on the current time and on the operator defining the right-hand side of the evolution equation, we use the notation

$$
u(t)=\mathcal{E}_{A+\varepsilon B}(t) u(0), \quad t \in\left[0, \frac{1}{\varepsilon} T\right]
$$

We employ the following hypotheses on the operators defining (2.1). For any exponent $\vartheta \geq 0$, we denote by $X_{\vartheta}=D\left(A^{\vartheta}\right) \subseteq X$ the fractional power spaces associated with $A$; in particular, the relations $X_{0}=X$ and $X_{1}=D(A)$ hold.

\section{Hypothesis 2.1.}

(i) Assume that the unbounded linear operator $A: D(A) \rightarrow X$ generates a strongly continuous group of isometries $\left(\mathcal{E}_{A}(t)\right)_{t \in \mathbb{R}}$ on the underlying Banach space and that the associated propagator is $T_{0}$-periodic for some $T_{0}>0$

$$
\mathcal{E}_{A}\left(T_{0}\right)=I
$$

Suppose further that the evolution operator preserves the norm on any fractional power space

$$
\left\|\mathcal{E}_{A}(t)\right\|_{X_{\vartheta} \leftarrow X_{\vartheta}}=1, \quad t \in \mathbb{R}, \quad \vartheta \geq 0 .
$$

(ii) Assume that the linear operator $B: X \rightarrow X$ is bounded, that is, the following estimate is satisfied for $\vartheta_{0}=0$ with some constant $C_{B, 0}>0$

$$
\|B v\|_{X_{\vartheta_{0}}} \leq C_{B, \vartheta_{0}}\|v\|_{X_{\vartheta_{0}}}, \quad v \in X_{\vartheta_{0}} .
$$

(iii) Assume that the linear operator $A+\varepsilon B: D(A) \rightarrow X$ generates a group of isometries on $X$.

\section{Remark.}

(a) The boundedness of $B: X \rightarrow X$ in particular ensures that $A+\varepsilon B: D(A) \rightarrow X$ generates a strongly continuous group on $X$.

(b) The statement of Theorem 3.5 remains valid when replacing hypothesis (iii) with the requirement

$$
\left\|\mathcal{E}_{A+\varepsilon B}(t)\right\|_{X \leftarrow X} \leq \mathrm{e}^{C t}, \quad t \in \mathbb{R}
$$

for some constant $C>0$.

(c) Provided that the considered potential is sufficiently regular, it is also justified to require the bound (2.2c) to hold for certain integer exponents $\vartheta_{0}>0$, see Section 2.2; this additional assumption will be used in the derivation of our main result. 


\subsection{Model equation}

\subsubsection{Model equation}

The general hypotheses on (2.1) are according to time-dependent linear Schrödinger equations involving a selfadjoint differential operator and a regular real-valued potential

$$
\mathrm{i} \partial_{t} \psi(x, t)=\mathcal{A}(x) \psi(x, t)+\varepsilon V(x) \psi(x, t), \quad(x, t) \in \Omega \times\left(0, \frac{1}{\varepsilon} T\right] ;
$$

we focus on the practically most relevant case

$$
\mathcal{A}=-\Delta, \quad \Omega=\left(-a_{1}, a_{1}\right) \times \ldots \times\left(-a_{d}, a_{d}\right) \subset \mathbb{R}^{d},
$$

with $a_{\ell}>0$ for any $\ell \in\{1, \ldots, d\}$.

\subsubsection{Basic results}

As is well known, for our model equation, the eigenvalue relation

$$
\mathcal{A B}_{\mu}=\lambda_{\mu} \mathcal{B}_{\mu}, \quad \mu \in \mathbb{Z}^{d},
$$

holds with Fourier functions $\mathcal{B}_{\mu}: \mathbb{R}^{d} \rightarrow \mathbb{C}$ and corresponding non-negative eigenvalues given by

$$
\mathcal{B}_{\mu}(x)=\prod_{\ell=1}^{d} \frac{1}{\sqrt{2 a_{\ell}}} \mathrm{e}^{\mathrm{i} \mu_{\ell} \pi\left(x_{\ell} / a_{\ell}+1\right)}, \quad \lambda_{\mu}=\pi^{2} \sum_{\ell=1}^{d} \frac{\mu_{\ell}^{2}}{a_{\ell}^{2}} \geq 0
$$

where $x=\left(x_{1}, \ldots, x_{d}\right) \in \mathbb{R}^{d}$ and $\mu=\left(\mu_{1}, \ldots, \mu_{d}\right) \in \mathbb{Z}^{d}$; evidently, the Fourier functions satisfy periodic boundary conditions on $\bar{\Omega}$. Making use of the fact that the Fourier functions form a complete orthonormal system

$$
\left(\mathcal{B}_{\mu} \mid \mathcal{B}_{\widetilde{\mu}}\right)_{L^{2}}=\delta_{\mu \widetilde{\mu}}, \quad \mu, \widetilde{\mu} \in \mathbb{Z}^{d}
$$

in the Hilbert space $L^{2}(\Omega)$, the spectral representation

$$
v=\sum_{\mu \in \mathbb{Z}^{d}} c_{\mu}(v) \mathcal{B}_{\mu}, \quad c_{\mu}(v)=\left(v \mid \mathcal{B}_{\mu}\right)_{L^{2}}, \quad \mu \in \mathbb{Z}^{d}, \quad v \in L^{2}(\Omega),
$$

follows, and by Parseval's identity the relation

$$
\|v\|_{L^{2}}^{2}=\sum_{\mu \in \mathbb{Z}^{d}}\left|c_{\mu}(v)\right|^{2}, \quad v \in L^{2}(\Omega)
$$

holds. By means of the spectral decomposition (2.4c) and the eigenvalue relation (2.4a), the representation

$$
\mathcal{E}_{-\mathrm{i} \mathcal{A}}(t) v=\sum_{\mu \in \mathbb{Z}^{d}} c_{\mu}(v) \mathrm{e}^{-\mathrm{i} t \lambda_{\mu}} \mathcal{B}_{\mu}, \quad t \in \mathbb{R}, \quad v \in L^{2}(\Omega),
$$

is obtained. For any exponent $\vartheta \geq 0$, the fractional power space

$$
X_{\vartheta}=\left\{v \in L^{2}(\Omega):\left\|\mathcal{A}^{\vartheta} v\right\|_{L^{2}}^{2}=\left\|\sum_{\mu \in \mathbb{Z}^{d}} c_{\mu}(v) \lambda_{\mu}^{\vartheta} \mathcal{B}_{\mu}\right\|_{L^{2}}^{2}=\sum_{\mu \in \mathbb{Z}^{d}}\left|c_{\mu}(v)\right|^{2} \lambda_{\mu}^{2 \vartheta}<\infty\right\}
$$

forms a Hilbert space with inner product and associated norm defined by

$$
\begin{gathered}
(v \mid w)_{X_{\vartheta}}=(v \mid w)_{L^{2}}+\left(\mathcal{A}^{\vartheta} v \mid \mathcal{A}^{\vartheta} w\right)_{L^{2}}, \quad v, w \in X_{\vartheta}, \\
\|v\|_{X_{\vartheta}}^{2}=\|v\|_{L^{2}}^{2}+\left\|\mathcal{A}^{\vartheta} v\right\|_{L^{2}}^{2}, \quad v \in X_{\vartheta},
\end{gathered}
$$

see also (2.4c), (2.4a), and (2.4d); especially, we have $X_{0}=L^{2}(\Omega)$ and $D(\mathcal{A})=X_{1}$. 


\subsubsection{Verification of hypotheses}

The model problem (2.3) corresponds to an evolutionary Schrödinger equation on the Hilbert space $X=$ $L^{2}(\Omega)$, with $A=-\mathrm{i} \mathcal{A}=\mathrm{i} \Delta: X_{1} \rightarrow X$ and operator $B=-\mathrm{i} V: X \rightarrow X$, acting as a multiplication operator. In the present situation, it is straightforward to justify the requirements of Hypothesis 2.1; as usual in a Hilbert space setting, we use the notion unitary operator instead of isometry.

(i) (a) Stone's Theorem ensures that $A$ is the infinitesimal generator of a strongly continuous one-parameter family $\left(\mathcal{E}_{A}(t)\right)_{t \in \mathbb{R}}$ of unitary operators (see [18], Chaps. 1,7); in particular, the first relation in (2.2a) follows from the solution representation (2.4e) and Parseval's identity (2.4d).

(b) Provided that the ratios of the positive real numbers defining the spatial domain $\Omega \subset \mathbb{R}^{d}$ are rational, the propagator $\mathcal{E}_{A}(\cdot)$ is periodic in time. More precisely, whenever the relation $r_{\ell} a_{\ell}^{2}=a_{1}^{2}$ holds with $r_{\ell} \in \mathbb{Q}$ for any integer $\ell$ such that $2 \leq \ell \leq d$, there exists a positive integer $\nu \in \mathbb{N}$ such that

$$
k_{\mu}=\nu \sum_{\ell=1}^{d} r_{\ell} \mu_{\ell}^{2} \in \mathbb{N}
$$

for every $\mu \in \mathbb{Z}^{d}$; setting $T_{0}=\frac{2}{\pi} a_{1}^{2} \nu$ yields

$$
T_{0} \lambda_{\mu}=T_{0} \pi^{2} \sum_{\ell=1}^{d} \frac{\mu_{\ell}^{2}}{a_{\ell}^{2}}=2 \pi k_{\mu}
$$

which implies $\mathrm{e}^{-\mathrm{i} T_{0} \lambda_{\mu}}=1$ for all $\mu \in \mathbb{Z}^{d}$ or equivalently $\mathcal{E}_{A}\left(T_{0}\right)=I$, see (2.4e). Especially, whenever $a_{\ell}=\pi$ for all $\ell \in\{1, \ldots, d\}$, the propagator $\mathcal{E}_{A}(\cdot)$ is $2 \pi$-periodic.

(c) For any exponent $\vartheta>0$ the spectral decomposition (2.4c), the solution representation (2.4e), the eigenvalue relation (2.4a), and Parseval's identity (2.4d) imply

$$
\left\|A^{\vartheta} \mathcal{E}_{A}(t) v\right\|_{L^{2}}^{2}=\left\|\sum_{\mu \in \mathbb{Z}^{d}} c_{\mu}(v) \mathrm{e}^{-\mathrm{i} t \lambda_{\mu}} \lambda_{\mu}^{\vartheta} \mathcal{B}_{\mu}\right\|_{L^{2}}^{2}=\left\|A^{\vartheta} v\right\|_{L^{2}}^{2}, \quad v \in X_{\vartheta}, \quad t \in \mathbb{R}
$$

which further yields

$$
\begin{aligned}
\left\|\mathcal{E}_{A}(t)\right\|_{X_{\vartheta} \leftarrow X_{\vartheta}} & =\sup _{\|v\|_{X_{\vartheta}=1}}\left\|\mathcal{E}_{A}(t) v\right\|_{X_{\vartheta}}=\sup _{\|v\|_{X_{\vartheta}=1}}\left(\left\|\mathcal{E}_{A}(t) v\right\|_{L^{2}}^{2}+\left\|A^{\vartheta} \mathcal{E}_{A}(t) v\right\|_{L^{2}}^{2}\right)^{\frac{1}{2}} \\
& =\sup _{\|v\|_{X_{\vartheta}=1}}\left(\|v\|_{L^{2}}^{2}+\left\|A^{\vartheta} v\right\|_{L^{2}}^{2}\right)^{\frac{1}{2}}=\sup _{\|v\|_{X_{\vartheta}}=1}\|v\|_{X_{\vartheta}}=1,
\end{aligned}
$$

see also $(2.4 \mathrm{~g})$ for the definition of the norm in the fractional power space $X_{\vartheta}$.

(ii) (a) Provided that the potential satisfies $V \in \mathcal{C}(\bar{\Omega})$, the estimate

$$
\|B v\|_{L^{2}} \leq C_{B, 0}\|v\|_{L^{2}}, \quad v \in L^{2}(\Omega),
$$

follows at once with $C_{B, 0}=\|V\|_{\mathcal{C}(\bar{\Omega})}$.

(b) Assuming that $V \in \mathcal{C}^{2 \vartheta_{0}}(\bar{\Omega})$ holds for some integer $\vartheta_{0}>0$, our aim is to deduce a bound for

$$
\|B v\|_{X_{\vartheta_{0}}}=\left(\|B v\|_{L^{2}}^{2}+\left\|A^{\vartheta_{0}}(B v)\right\|_{L^{2}}^{2}\right)^{\frac{1}{2}}
$$

For any multi-index $\kappa=\left(\kappa_{1}, \ldots, \kappa_{d}\right) \in \mathbb{N}^{d}$ we set $|\kappa|=\kappa_{1}+\ldots+\kappa_{d}$ as well as $\partial_{x}^{\kappa}=\partial_{x_{1}}^{\kappa_{1}} \ldots \partial_{x_{d}}^{\kappa_{d}}$. Straightforward differentiation by means of the Leibniz rule shows that $A^{\vartheta_{0}}(B v)=-\mathrm{i}^{\vartheta_{0}+1} \Delta^{\vartheta_{0}}(V v), \vartheta_{0} \in \mathbb{N}$, 
comprises terms of the form $\partial_{x}^{\kappa-\widetilde{\kappa}} V \partial_{x}^{\widetilde{\kappa}} v$ with $\kappa, \widetilde{\kappa} \in \mathbb{N}^{d}$ such that $|\kappa|=2 \vartheta_{0}$ and $\widetilde{\kappa} \leq \kappa$, componentwise. The representation (2.4c) together with the eigenvalue relation (2.4a) imply the estimate

$$
\left\|\partial_{x}^{\kappa} v\right\|_{L^{2}} \leq\left\|A^{\frac{1}{2}|\kappa|} v\right\|_{L^{2}}, \quad v \in X_{\frac{1}{2}|\kappa|}, \quad \kappa \in \mathbb{N}^{d} .
$$

As a consequence, the relation

$$
\|B v\|_{X_{\vartheta_{0}}} \leq C_{B, \vartheta_{0}}\|v\|_{X_{\vartheta_{0}}}, \quad v \in X_{\vartheta_{0}}
$$

follows with constant $C_{B, \vartheta_{0}}>0$ depending on the bounds for the derivatives of the potential $V$ up to order $2 \vartheta_{0}$.

(iii) The unitarity of the evolution operator follows from Stone's Theorem.

\subsubsection{Extensions}

Making use of the fact that the considered differential operator $\mathcal{A}: D(\mathcal{A}) \rightarrow X$ is self-adjoint and positive semi-definite with pure point spectrum, permits to incorporate relevant problems of the form (2.3a) that are related to other spectral methods such as the Hermite or generalised Laguerre-Fourier-Hermite spectral method, see $[13,16]$ and references therein. In this situation, standard results [20] ensure that the family of eigenfunctions forms a countable complete orthonormal system in the underlying Hilbert space.

\subsection{Time discretisation}

In this section, we introduce the general format of multi-revolution composition time-splitting methods for the numerical solution of time-dependent highly oscillatory Schrödinger equations; for this purpose, it is convenient to employ the compact formulation as abstract evolution equation (2.1).

\subsubsection{Exact solution values}

Throughout, we suppose that the final time is an integer multiple of the period

$$
\frac{1}{\varepsilon} T=N N_{0} T_{0}, \quad N, N_{0} \in \mathbb{N}_{\geq 1},
$$

see also (2.2a); we note that the size of the increment

$$
0<H=\varepsilon N_{0}<1
$$

effects the quality of the numerical approximation. Clearly, the identity

$$
T=N H T_{0}
$$

holds. The aim is to determine numerical approximations to the exact solution values

$$
\widehat{u}_{n}=u\left(n N_{0} T_{0}\right), \quad n \in\{1, \ldots, N\} .
$$

\subsubsection{Approximation by composition}

In a first step, we apply a multi-revolution composition method of order $P \in \mathbb{N}_{>1}$, defined by real coefficients $\left(\alpha_{j}, \beta_{j}\right)_{j=1}^{r}$. The resulting approximations require the evaluation of certain exact evolution operators associated with different right-hand sides

$$
\begin{aligned}
u_{n+1} & =\mathcal{C}_{A+\varepsilon B}\left(N_{0} T_{0}\right) u_{n} \approx \widehat{u}_{n+1}=\mathcal{E}_{A+\varepsilon B}\left(N_{0} T_{0}\right) \widehat{u}_{n}, \\
\mathcal{C}_{A+\varepsilon B}\left(N_{0} T_{0}\right) & =\prod_{j=1}^{r}\left(\mathcal{E}_{A-\beta_{j} \varepsilon N_{0} B}\left(-T_{0}\right) \mathcal{E}_{A+\alpha_{j} \varepsilon N_{0} B}\left(T_{0}\right)\right),
\end{aligned}
$$

where $n \in\{0, \ldots, N-1\}$, 


\subsubsection{Time discretisation}

For the time discretisation of (2.1), the composition approach (2.7) is combined with an exponential operator splitting method of order $p \in \mathbb{N}_{\geq 1}$, defined by real coefficients $\left(a_{k}, b_{k}\right)_{k=1}^{s}$. More precisely, for the approximation of $\mathcal{E}_{A+\gamma B}\left(T_{0}\right)$ a splitting method with time stepsize $h=\frac{T_{0}}{K}>0$ for some $K \in \mathbb{N}_{\geq 1}$ is applied

$$
\begin{aligned}
& \mathcal{S}_{A+\gamma B}^{K}\left(T_{0}\right)=\left(\mathcal{S}_{A+\gamma B}(h)\right)^{K} \approx \mathcal{E}_{A+\gamma B}\left(T_{0}\right), \\
& \mathcal{S}_{A+\gamma B}(h)=\prod_{\ell=1}^{s}\left(\mathcal{E}_{\gamma B}\left(b_{\ell} h\right) \mathcal{E}_{A}\left(a_{\ell} h\right)\right) \approx \mathcal{E}_{A+\gamma B}(h),
\end{aligned}
$$

and analogously for the computation of $\mathcal{E}_{A-\gamma B}\left(-T_{0}\right)$. This yields the following relation involving the timediscrete evolution operator

$$
\begin{aligned}
v_{n+1} & =\mathcal{D}_{A+\varepsilon B}\left(N_{0} T_{0}\right) v_{n} \approx u_{n+1}=\mathcal{C}_{A+\varepsilon B}\left(N_{0} T_{0}\right) u_{n}, \\
\mathcal{D}_{A+\varepsilon B}\left(N_{0} T_{0}\right) & =\prod_{j=1}^{r}\left(\mathcal{S}_{A-\beta_{j} \varepsilon N_{0} B}^{K}\left(-T_{0}\right) \mathcal{S}_{A+\alpha_{j} \varepsilon N_{0} B}^{K}\left(T_{0}\right)\right)
\end{aligned}
$$

where $n \in\{0, \ldots, N-1\}$.

\subsubsection{Initial approximation}

We suppose $v_{0} \approx \widehat{u}_{0}=u(0)$ to be a suitably chosen initial approximation for (2.8) and set $u_{0}=v_{0}$ in $(2.7)$.

\section{Convergence Analysis}

In this section, we deduce our main result on the convergence behaviour of multi-revolution composition timesplitting methods applied to highly oscillatory evolution equations of Schrödinger type. The considered class of time discretisations inherits the favourable properties of the underlying methods in regard to stability, accuracy, efficiency, and the preservation of physically relevant quantities. Essential prerequisites for the estimation of the global error are stability estimates and bounds for the defects. Due to the fact that the arising evolution operators are isometries, it is straighforward to establish stability results with respect to the underlying Banach space, see Section 3.1. A fundamental error estimate for high-order multi-revolution composition methods is stated in Section 3.2, and a result explaining the improved error behaviour of splitting methods is given in Section 3.3.

\subsection{Stability}

\subsubsection{Isometry}

We make use of the hypothesis that the exact evolution operator associated with the linear operator $A+\gamma B$ defines an isometry on the underlying Banach space

$$
\left\|\mathcal{E}_{A+\gamma B}(t) v\right\|_{X}=\|v\|_{X}, \quad v \in X, \quad t \in \mathbb{R}, \quad \gamma \in \mathbb{R},
$$

see Section 2.1 and 2.2 .

\subsubsection{Stability results}

The above relation at once implies

$$
\left\|\left(\mathcal{C}_{A+\varepsilon B}\left(N_{0} T_{0}\right)\right)^{\ell} v\right\|_{X}=\|v\|_{X}, \quad\left\|\left(\mathcal{D}_{A+\varepsilon B}\left(N_{0} T_{0}\right)\right)^{\ell} v\right\|_{X}=\|v\|_{X},
$$

for any $v \in X$ and for all integers $\ell \in \mathbb{N}$, see also (2.7) and (2.8). 


\subsection{Error bounds for MRCMs}

In this section, we study the approximation error of multi-revolution composition methods for evolution equations of Schrödinger type; in particular, we aim at an error estimate of the form

$$
\left\|\mathcal{C}_{A+\varepsilon B}\left(N_{0} T_{0}\right) v-\mathcal{E}_{A+\varepsilon B}\left(N_{0} T_{0}\right) v\right\|_{X} \leq C H^{P+1}\|v\|_{X}
$$

showing that the increment $0<H=\varepsilon N_{0}<1$ and the order $P \in \mathbb{N}_{\geq 1}$ of the composition method determine the approximation quality.

\subsubsection{Auxiliary result}

A fundamental auxiliary result used in the derivation of such an error estimate ensures that the evolution operator over one period and a related operator are near-identity smooth maps with respect to $\varepsilon \in \mathbb{R}$ and provides bounds for their derivatives.

Lemma 3.1. The evolution operator over one period and its reverse evolution

$$
\begin{aligned}
& \mathbb{R} \longrightarrow \mathcal{L}(X): \varepsilon \longmapsto \Phi_{\varepsilon}=\mathcal{E}_{A+\varepsilon B}\left(T_{0}\right), \\
& \mathbb{R} \longrightarrow \mathcal{L}(X): \varepsilon \longmapsto \Phi_{-\varepsilon}^{-1}=\mathcal{E}_{A-\varepsilon B}\left(-T_{0}\right),
\end{aligned}
$$

are near-identity smooth maps, satisfying $\Phi_{0}=I=\Phi_{0}^{-1}$ and the bounds

$$
\left\|\partial_{\varepsilon}^{n} \Phi_{\varepsilon} v\right\|_{X} \leq\left(C_{B, 0} T_{0}\right)^{n}\|v\|_{X}, \quad\left\|\partial_{\varepsilon}^{n} \Phi_{-\varepsilon}^{-1} v\right\|_{X} \leq\left(C_{B, 0} T_{0}\right)^{n}\|v\|_{X}, \quad n \in \mathbb{N} .
$$

Proof. Let $\varepsilon, t \in \mathbb{R}, n \in \mathbb{N}_{\geq 1}$, and $v \in X$. The linear variation-of-constants formula reads

$$
\mathcal{E}_{A+\varepsilon B}(t) v=\mathcal{E}_{A}(t) v+\int_{0}^{t} \mathcal{E}_{A}(t-\tau) \varepsilon B \mathcal{E}_{A+\varepsilon B}(\tau) v \mathrm{~d} \tau
$$

and hence differentiation with respect to $\varepsilon$ yields

$$
\mathcal{U}(t)=\partial_{\varepsilon}^{n} \mathcal{E}_{A+\varepsilon B}(t) v=\int_{0}^{t} \mathcal{E}_{A}(t-\tau)\left(\varepsilon B \mathcal{U}(\tau)+n B \partial_{\varepsilon}^{n-1} \mathcal{E}_{A+\varepsilon B}(\tau) v\right) \mathrm{d} \tau .
$$

Making use of the fact that this is just the representation by the variation-of-constants formula for the solution to the initial value problem

$$
\left\{\begin{array}{l}
\mathcal{U}^{\prime}(t)=A \mathcal{U}(t)+\varepsilon B \mathcal{U}(t)+n B \partial_{\varepsilon}^{n-1} \mathcal{E}_{A+\varepsilon B}(t) v, \quad t \in \mathbb{R} \\
\mathcal{U}(0)=0
\end{array}\right.
$$

the following relation

$$
\mathcal{U}(t)=\partial_{\varepsilon}^{n} \mathcal{E}_{A+\varepsilon B}(t) v=n \int_{0}^{t} \mathcal{E}_{A+\varepsilon B}(t-\tau) B \partial_{\varepsilon}^{n-1} \mathcal{E}_{A+\varepsilon B}(\tau) v \mathrm{~d} \tau
$$

and, by an induction argument, the bound

$$
\left\|\partial_{\varepsilon}^{n} \mathcal{E}_{A+\varepsilon B}(t) v\right\|_{X} \leq\left(C_{B, 0} t\right)^{n}\|v\|_{X}
$$

is obtained. Indeed, assuming that the claimed result holds true at step $n-1$, the above representation and the fact that $\mathcal{E}_{A+\varepsilon B}(\cdot)$ is an isometry yield

$$
\begin{aligned}
\left\|\partial_{\varepsilon}^{n} \mathcal{E}_{A+\varepsilon B}(t) v\right\|_{X} & \leq n \int_{0}^{t}\left\|B \partial_{\varepsilon}^{n-1} \mathcal{E}_{A+\varepsilon B}(\tau) v\right\|_{X} \mathrm{~d} \tau \leq n C_{B, 0} \int_{0}^{t}\left\|\partial_{\varepsilon}^{n-1} \mathcal{E}_{A+\varepsilon B}(\tau) v\right\|_{X} \mathrm{~d} \tau \\
& \leq n C_{B, 0} \int_{0}^{t}\left(C_{B, 0} \tau\right)^{n-1} \mathrm{~d} \tau\|v\|_{X}=\left(C_{B, 0} t\right)^{n}\|v\|_{X} .
\end{aligned}
$$

Finally, setting $t=T_{0}$ or $t=-T_{0}$, respectively, proves the assertion. 
Theorem 3.2. Under the requirements of Hypothesis 2.1, a multi-revolution composition method of the form (2.7) applied to the evolution equation (2.1) fulfills the error estimate

$$
\left\|\mathcal{C}_{A+\varepsilon B}\left(N_{0} T_{0}\right) v-\mathcal{E}_{A+\varepsilon B}\left(N_{0} T_{0}\right) v\right\|_{X} \leq \frac{C}{(P+1) !} C_{B, 0}^{P+1} T_{0}^{P+1} H^{P+1}\|v\|_{X}, \quad v \in X
$$

provided that the coefficients satisfy the (nonstiff) conditions for order $P \in \mathbb{N}_{\geq 1}$.

Proof. Our proof in the lines of [7] is based on the fact that the flow map associated with a multi-revolution composition method can be written as $\Phi_{\varepsilon} v=v+\varepsilon \Phi_{\varepsilon}^{(1)} v$, where $\Phi_{\varepsilon}^{(1)}$ is smooth with respect to $\varepsilon \in \mathbb{R}$; indeed, in the present situation, the evolution operator over one period can be cast into this form

$$
\Phi_{\varepsilon} v=\mathcal{E}_{A+\varepsilon B}\left(T_{0}\right) v=v+\varepsilon \int_{0}^{T_{0}} \mathcal{E}_{A}\left(T_{0}-\tau\right) B \mathcal{E}_{A+\varepsilon B}(\tau) v \mathrm{~d} \tau
$$

for any $v \in X$, see the proof of Lemma 3.1. Employing the abbreviation

$$
\Psi_{H}=\prod_{j=1}^{r}\left(\Phi_{-\beta_{j} H}^{-1} \Phi_{\alpha_{j} H}\right), \quad H=\varepsilon N_{0},
$$

the approximation error takes the form

$$
\mathcal{C}_{A+\varepsilon B}\left(N_{0} T_{0}\right)-\mathcal{E}_{A+\varepsilon B}\left(N_{0} T_{0}\right)=\Psi_{H}-\Phi_{H / N_{0}}^{N_{0}}
$$

We perform Taylor expansions of $\Psi_{H}$ and $\Phi_{H / N_{0}}^{N_{0}}$ with respect to the increment $H$; we note that, by construction, the validity of the order conditions ensure that the leading contributions in these expansions coincide such that

$$
\Psi_{H}-\Phi_{H / N_{0}}^{N_{0}}=\frac{1}{P !} \int_{0}^{H}(H-\tau)^{P}\left(\partial_{\tau}^{P+1} \Psi_{\tau}-\partial_{\tau}^{P+1} \Phi_{\tau / N_{0}}^{N_{0}}\right) \mathrm{d} \tau
$$

see [7]. Thus, it remains to estimate the $(P+1)$-st derivatives of the mappings $\tau \mapsto \Psi_{\tau}$ and $\tau \mapsto \Phi_{\tau / N_{0}}^{N_{0}}$. On the one hand, by the chain rule we obtain

$$
\partial_{\varepsilon}^{P+1} \Phi_{\varepsilon}^{N_{0}}=\sum_{m_{1}+\ldots+m_{N_{0}}=P+1} \frac{(P+1) !}{m_{1} ! \ldots m_{N_{0}} !} \partial_{\varepsilon}^{m_{1}} \Phi_{\varepsilon} \ldots \partial_{\varepsilon}^{m_{N_{0}}} \Phi_{\varepsilon},
$$

which by Lemma 3.1 further implies

$$
\left\|\partial_{\varepsilon}^{P+1} \Phi_{\varepsilon}^{N_{0}} v\right\|_{X} \leq \sum_{m_{1}+\ldots+m_{N_{0}}=P+1} \frac{(P+1) !}{m_{1} ! \ldots m_{N_{0}} !}\left(C_{B, 0} T_{0}\right)^{P+1}\|v\|_{X} \leq\left(C_{B, 0} N_{0} T_{0}\right)^{P+1}\|v\|_{X} ;
$$

a change of variable yields

$$
\left\|\partial_{\tau}^{P+1} \Phi_{\tau / N_{0}}^{N_{0}} v\right\|_{X} \leq\left(C_{B, 0} T_{0}\right)^{P+1}\|v\|_{X} .
$$

Similar arguments lead to the relation

$$
\begin{aligned}
\partial_{\tau}^{P+1} \Psi_{\tau} & =\sum_{m_{1}+\ldots+m_{2 r}=P+1} \frac{(P+1) !}{m_{1} ! \ldots m_{2 r} !} \partial_{\tau}^{m_{1}} \Phi_{-\beta_{1} \tau}^{-1} \ldots \partial_{\tau}^{m_{2 r}} \Phi_{\alpha_{r} \tau} \\
& =\sum_{m_{1}+\ldots+m_{2 r}=P+1} \frac{(P+1) !}{m_{1} ! \ldots m_{2 r} !} \beta_{1}^{m_{1}} \ldots \alpha_{r}^{m_{2 r}} \partial_{\varepsilon}^{m_{1}} \Phi_{-\beta_{1} \tau}^{-1} \ldots \partial_{\varepsilon}^{m_{2 r}} \Phi_{\alpha_{r} \tau}
\end{aligned}
$$


which by the validity of the first-order conditions implies the estimate

$$
\left\|\partial_{\tau}^{P+1} \Psi_{\tau} v\right\|_{X} \leq\left(\left|\alpha_{1}\right|+\ldots+\left|\alpha_{r}\right|+\left|\beta_{1}\right|+\ldots+\left|\beta_{r}\right|\right)^{P+1}\left(C_{B, 0} T_{0}\right)^{P+1}\|v\|_{X} \leq C\left(C_{B, 0} T_{0}\right)^{P+1}\|v\|_{X} ;
$$

we note that the coefficients defining the multi-revolution composition method, which in general depend on $N_{0}$, are bounded, see [7]. Altogether this leads to the bound

$$
\left\|\Psi_{H} v-\Phi_{H / N_{0}}^{N_{0}} v\right\|_{X} \leq \frac{C}{P !}\left(C_{B, 0} T_{0}\right)^{P+1} \int_{0}^{H} \tau^{P} \mathrm{~d} \tau\|v\|_{X} \leq \frac{C}{(P+1) !} C_{B, 0}^{P+1} T_{0}^{P+1} H^{P+1}\|v\|_{X},
$$

which concludes the proof.

\subsection{Error bounds for splitting methods}

In this section, we analyse the approximation error due to the application of splitting methods for the realisation of multi-revolution composition methods.

\subsubsection{Notation and telescopic identity}

For the following considerations it is convenient to employ the abbreviations $\Phi_{\gamma, h}=\mathcal{S}_{A+\gamma B}^{K}\left(T_{0}\right), \Phi_{\gamma}=$ $\mathcal{E}_{A+\gamma B}\left(T_{0}\right)$, and $\Phi_{-\gamma}^{-1}=\mathcal{E}_{A-\gamma B}\left(-T_{0}\right)$ for $\gamma \in \mathbb{R} ;$ thus, the error takes the form

$$
\mathcal{D}_{A+\varepsilon B}\left(N_{0} T_{0}\right)-\mathcal{C}_{A+\varepsilon B}\left(N_{0} T_{0}\right)=\prod_{j=1}^{r}\left(\Phi_{-\beta_{j} H,-h} \Phi_{\alpha_{j} H, h}\right)-\prod_{j=1}^{r}\left(\Phi_{-\beta_{j} H}^{-1} \Phi_{\alpha_{j} H}\right) .
$$

By means of a telescopic identity the difference reads

$$
\begin{aligned}
\mathcal{D}_{A+\varepsilon B}\left(N_{0} T_{0}\right)-\mathcal{C}_{A+\varepsilon B}\left(N_{0} T_{0}\right)= & \sum_{\ell=1}^{r}\left(\prod_{j=1}^{\ell-1}\left(\Phi_{-\beta_{j} H,-h} \Phi_{\alpha_{j} H, h}\right)\right) \Phi_{-\beta_{\ell} H,-h}\left(\Phi_{\alpha_{\ell} H, h}-\Phi_{\alpha_{\ell} H}\right) \\
& \times\left(\prod_{k=\ell+1}^{r}\left(\Phi_{-\beta_{k} H}^{-1} \Phi_{\alpha_{k} H}\right)\right) \\
& +\sum_{\ell=1}^{r}\left(\prod_{j=1}^{\ell-1}\left(\Phi_{-\beta_{j} H,-h} \Phi_{\alpha_{j} H, h}\right)\right)\left(\Phi_{-\beta_{\ell} H,-h}-\Phi_{-\beta_{\ell} H}^{-1}\right) \Phi_{\alpha_{\ell} H} \\
& \times\left(\prod_{k=\ell+1}^{r}\left(\Phi_{-\beta_{k} H}^{-1} \Phi_{\alpha_{k} H}\right)\right) .
\end{aligned}
$$

\subsubsection{Auxiliary expansion}

The following auxiliary result provides an expansion for the error of a time-splitting method over one period; this result is adapted from [8], where the corresponding estimate was proven in the nonlinear case.

Lemma 3.3. Consider a splitting method of order $p \in \mathbb{N}_{\geq 1}$ for the time integration of the highly oscillatory linear evolution equation (2.1) over a single period. Provided that the time stepsize $h>0$ satisfies the condition $T_{0} / h \in \mathbb{N}$, the decomposition

$$
\Phi_{\varepsilon, h}-\Phi_{\varepsilon}=\varepsilon S_{h}+\varepsilon^{2} T_{h}+\varepsilon^{3} r_{\varepsilon, h}
$$

holds; the operators $S_{h}, T_{h}$ are independent of $\varepsilon \in \mathbb{R}$, and for all $\sigma \in \mathbb{N}$ the following relations are valid

$$
\begin{array}{rll}
\forall m \in \mathbb{N} \quad \forall v \in X_{(\sigma+m) / 2}: & \left\|S_{h} v\right\|_{X_{\sigma / 2}} \leq C h^{m}\|v\|_{X_{(\sigma+m) / 2}}, \\
\forall q \in \mathbb{N} \text { with } q \leq p \quad \forall v \in X_{(\sigma+q) / 2}: & \left\|T_{h} v\right\|_{X_{\sigma / 2}} \leq C h^{q}\|v\|_{X_{(\sigma+q) / 2}}, \\
\forall q \in \mathbb{N} \text { with } q \leq p \quad \forall v \in X_{(\sigma+q) / 2}: & \left\|r_{\varepsilon, h} v\right\|_{X_{\sigma / 2}} \leq C h^{q}\|v\|_{X_{(\sigma+q) / 2}} .
\end{array}
$$




\subsubsection{Error estimate}

With the help of the telescopic identity and the auxiliary result the following error estimate is obtained.

Theorem 3.4. Under the requirements of Hypothesis 2.1 on the highly oscillatory evolution equation (2.1), a multi-revolution composition method combined with a time-splitting method of order $p \in \mathbb{N}_{\geq 1}$ fulfills the error estimate

$$
\left\|\mathcal{D}_{A+\varepsilon B}\left(N_{0} T_{0}\right) v-\mathcal{C}_{A+\varepsilon B}\left(N_{0} T_{0}\right) v\right\|_{X} \leq C H h^{m}\|v\|_{X_{m / 2}}+C H^{2} h^{p}\|v\|_{X_{p / 2}}
$$

provided that the multi-revolution composition method is of order $P \in \mathbb{N}_{\geq 2}$ and the splitting method is symmetric, the improved bound

$$
\left\|\mathcal{D}_{A+\varepsilon B}\left(N_{0} T_{0}\right) v-\mathcal{C}_{A+\varepsilon B}\left(N_{0} T_{0}\right) v\right\|_{X} \leq C H h^{m}\|v\|_{X_{m / 2}}+C \varepsilon H h^{p}\|v\|_{X_{p / 2}}
$$

is valid.

Proof.

(i) Non-symmetric splitting methods. From Lemma 3.3 one deduces that, for all $m \in \mathbb{N}^{*}$, for all $\ell$ and for all $v \in X_{m / 2} \cap X_{p / 2}$, we have

$$
\left\|\Phi_{\alpha_{\ell} H, h} v-\Phi_{\alpha_{\ell} H} v\right\|_{X}+\left\|\Phi_{-\beta_{\ell} H,-h} v-\Phi_{-\beta_{\ell} H}^{-1} v\right\|_{X} \leq C H h^{m}\|v\|_{X_{m / 2}}+C H^{2} h^{p}\|v\|_{X_{p / 2}} .
$$

Here we used that the coefficients $\alpha_{\ell}$ and $\beta_{\ell}$ are uniformly bounded with respect to $\varepsilon, h$ and $N_{0}$. Inserting this estimate into the telescopic identity and using the uniform boundedness of $\Phi_{\varepsilon}$ and $\Phi_{\varepsilon, h}$ on fractional power spaces, yields the stated bound for non-symmetric splitting methods.

(ii) Symmetric splitting methods. Let $\sigma \in \mathbb{N}$. If the underlying multi-revolution composition method at least of order two and the chosen splitting method is symmetric, the error estimate can be slightly improved. By means of the linear variation-of-constants formula

$$
\mathcal{E}_{A+\varepsilon B}\left(T_{0}\right)=I+\varepsilon \Psi_{\varepsilon}, \quad \Psi_{\varepsilon}=\int_{0}^{T_{0}} \mathcal{E}_{A}\left(T_{0}-\tau\right) B \mathcal{E}_{A+\varepsilon B}(\tau) \mathrm{d} \tau,
$$

we obtain the estimate

$$
\forall v \in X_{\sigma / 2}: \quad\left\|\Psi_{\varepsilon} v\right\|_{X_{\sigma / 2}}=\frac{1}{\varepsilon}\left\|\Phi_{\varepsilon} v-v\right\|_{X_{\sigma / 2}} \leq C\|v\|_{X_{\sigma / 2}}
$$

Similarly, using (3.3) together with the estimate of Lemma 3.3 with $m=q=0$, we get

$$
\forall v \in X_{\sigma / 2}: \quad\left\|\Psi_{\varepsilon, h} v\right\|_{X_{\sigma / 2}}=\frac{1}{\varepsilon}\left\|\Phi_{\varepsilon, h} v-v\right\|_{X_{\sigma / 2}} \leq C\|v\|_{X_{\sigma / 2}} .
$$

Consequently, applying again Lemma 3.3 with $q=p$ together with (3.3) and (3.4) and using the uniform boundedness of $\Phi_{\varepsilon}$ and $\Phi_{\varepsilon, h}$ on fractional power spaces, we deduce from the telescopic identity that

$$
\mathcal{D}_{A+\varepsilon B}\left(N_{0} T_{0}\right)-\mathcal{C}_{A+\varepsilon B}\left(N_{0} T_{0}\right)=\sum_{\ell=1}^{r}\left(\Phi_{\alpha_{\ell} H, h}-\Phi_{\alpha_{\ell} H}\right)+\sum_{\ell=1}^{r}\left(\Phi_{-\beta_{\ell} H,-h}-\Phi_{-\beta_{\ell} H}^{-1}\right)+A_{\varepsilon, h},
$$

where the remainder $A_{\varepsilon, h}$ is bounded for all $m \in \mathbb{N}^{*}$ and $v \in X_{m / 2} \cap X_{p / 2}$

$$
\left\|A_{\varepsilon, h} v\right\|_{X} \leq C H h^{m}\|v\|_{X_{m / 2}}+C H^{3} h^{p}\|v\|_{X_{p / 2}} .
$$

Next, we estimate the principal terms in (3.5). Lemma 3.3 yields

$$
\left\|\Phi_{\varepsilon, h} v-\Phi_{\varepsilon} v-\varepsilon^{2} T_{h} v\right\|_{X} \leq C|\varepsilon| h^{m}\|v\|_{X_{m / 2}}+|\varepsilon|^{3} h^{p}\|v\|_{X_{p / 2}} .
$$


We note that the time reversibility of the Schrödinger flow implies $\mathcal{E}_{A-\varepsilon B}\left(-T_{0}\right)=\left(\mathcal{E}_{A-\varepsilon B}\left(T_{0}\right)\right)^{-1}=\Phi_{-\varepsilon}^{-1}$ and that, for a symmetric splitting method, we have $\Phi_{\varepsilon,-h}=\Phi_{\varepsilon, h}^{-1}$. Employing the decompositions $\Phi_{-\varepsilon}^{-1}=I+\varepsilon \Psi_{-\varepsilon}^{-1}$ as well as $\Phi_{-\varepsilon,-h}=I-\varepsilon \Psi_{-\varepsilon,-h}$ and the resolvent formula, we thus have

$$
\begin{aligned}
\Phi_{-\varepsilon, h}^{-1}-\Phi_{-\varepsilon}^{-1} & =-\Phi_{-\varepsilon}^{-1}\left(\Phi_{-\varepsilon, h}-\Phi_{-\varepsilon}\right) \Phi_{-\varepsilon, h}^{-1}=-\Phi_{-\varepsilon}^{-1}\left(\Phi_{-\varepsilon, h}-\Phi_{-\varepsilon}\right) \Phi_{-\varepsilon,-h} \\
& =-\Phi_{-\varepsilon}^{-1}\left(-\varepsilon S_{h}+\varepsilon^{2} T_{h}-\varepsilon^{3} r_{-\varepsilon, h}\right) \Phi_{-\varepsilon,-h} \\
& =\varepsilon \Phi_{-\varepsilon}^{-1} S_{h} \Phi_{-\varepsilon,-h}+\varepsilon^{3} \Phi_{-\varepsilon}^{-1} r_{-\varepsilon, h} \Phi_{-\varepsilon,-h}-\left(I+\varepsilon \Psi_{-\varepsilon}^{-1}\right) \varepsilon^{2} T_{h}\left(I-\varepsilon \Psi_{-\varepsilon,-h}\right) \\
& =-\varepsilon^{2} T_{h}+\varepsilon \Phi_{-\varepsilon}^{-1} S_{h} \Phi_{-\varepsilon,-h}+\varepsilon^{3} \widetilde{r}_{\varepsilon, h}
\end{aligned}
$$

with remainder given by

$$
\widetilde{r}_{\varepsilon, h}=-\Psi_{-\varepsilon}^{-1} T_{h}+T_{h} \Psi_{-\varepsilon,-h}+\varepsilon \Psi_{-\varepsilon}^{-1} T_{h} \Psi_{-\varepsilon,-h}+\Phi_{-\varepsilon}^{-1} r_{-\varepsilon, h} \Phi_{-\varepsilon,-h}
$$

Hence, using the uniform boundedness of the operators $\Phi_{\varepsilon}, \Phi_{\varepsilon, h}, \Psi_{\varepsilon}$ and $\Psi_{-\varepsilon,-h}$ in fractional power spaces and the estimates given in Lemma 3.3, we obtain

$$
\left\|\Phi_{-\varepsilon,-h} v-\Phi_{-\varepsilon}^{-1} v+\varepsilon^{2} T_{h} v\right\|_{X} \leq C|\varepsilon| h^{m}\|v\|_{X_{m / 2}}+|\varepsilon|^{3} h^{p}\|v\|_{X_{p / 2}} .
$$

Finally, from (3.5)-(3.8), we deduce the decomposition

$$
\mathcal{D}_{A+\varepsilon B}\left(N_{0} T_{0}\right)-\mathcal{C}_{A+\varepsilon B}\left(N_{0} T_{0}\right)=\sum_{\ell=1}^{r}\left(\alpha_{\ell}^{2}-\beta_{\ell}^{2}\right) H^{2} T_{h}+B_{\varepsilon, h}
$$

with remainder satisfying the bound

$$
\left\|B_{\varepsilon, h} v\right\|_{X} \leq C H h^{m}\|v\|_{X_{m / 2}}+C H^{3} h^{p}\|v\|_{X_{p / 2}} .
$$

Provided that the underlying multi-revolution composition method is at least of order two, the order condition

$$
\sum_{\ell=1}^{r}\left(\alpha_{\ell}^{2}-\beta_{\ell}^{2}\right)=\frac{1}{N_{0}}
$$

is fulfilled, see [7]. Altogether, we finally obtain the stated error estimate.

\subsection{Convergence result}

In order to deduce a convergence estimate for the proposed time discretisations, our basic approach is to decompose the global error at the final time $\frac{1}{\varepsilon} T$ such that the building blocks reflect the stability properties and defects of the underlying methods. Interposing the approximation obtained by composition (2.7) yields $v_{N}-\widehat{u}_{N}=u_{N}-\widehat{u}_{N}+v_{N}-u_{N}$, see also (2.6). The repeated application of a telescopic identity leads to the global error representation

$$
\begin{aligned}
v_{N}-\widehat{u}_{N}= & \left(\mathcal{C}_{A+\varepsilon B}\left(N_{0} T_{0}\right)\right)^{N}\left(u_{0}-\widehat{u}_{0}\right) \\
& +\sum_{n=1}^{N}\left(\mathcal{C}_{A+\varepsilon B}\left(N_{0} T_{0}\right)\right)^{N-n}\left(\mathcal{C}_{A+\varepsilon B}\left(N_{0} T_{0}\right)-\mathcal{E}_{A+\varepsilon B}\left(N_{0} T_{0}\right)\right) \widehat{u}_{n-1} \\
& +\sum_{n=1}^{N}\left(\mathcal{D}_{A+\varepsilon B}\left(N_{0} T_{0}\right)\right)^{N-n}\left(\mathcal{D}_{A+\varepsilon B}\left(N_{0} T_{0}\right)-\mathcal{C}_{A+\varepsilon B}\left(N_{0} T_{0}\right)\right) u_{n-1}
\end{aligned}
$$

we recall that the error of the numerical initial value is captured by $v_{0} \approx \widehat{u}_{0}=u(0)$ and that $u_{0}=v_{0}$ holds by assumption. 


\subsubsection{Convergence result}

By means of the provided auxiliary results, we are able to establish the following convergence result.

Theorem 3.5 (Global error estimate). For the time discretisation of the linear Schrödinger equation (2.1) consider (2.8), defined by a multi-revolution composition method of nonstiff order $P \in \mathbb{N}_{\geq 1}$, applied with increment $0<H<1$, and a time-splitting method of nonstiff order $p \in \mathbb{N}_{\geq 1}$, applied with stepsize $0<h<1$. Under Hypothesis 2.1 with $\vartheta_{0} \geq \frac{p}{2}$ and the additional assumptions that the initial state $u_{0}$ and the exact solution values remain bounded in the fractional power space $X_{\vartheta_{0}}$, the following global error estimate holds

$$
\left\|v_{N}-u\left(\frac{1}{\varepsilon} T\right)\right\|_{X} \leq\left\|u_{0}-u(0)\right\|_{X}+C\left(H^{P}+h^{2 \vartheta_{0}}+H h^{p}\right)
$$

provided that the considered multi-revolution composition method is at least of order two and the splitting method is symmetric, the improved global error estimate

$$
\left\|v_{N}-u\left(\frac{1}{\varepsilon} T\right)\right\|_{X} \leq\left\|u_{0}-u(0)\right\|_{X}+C\left(H^{P}+h^{2 \vartheta_{0}}+\varepsilon h^{p}\right)
$$

is valid. The arising constant $C>0$ in particular depends on upper bounds for $\|V\|_{X_{\vartheta_{0}}}$ and $\max \left\{\|u(t)\|_{X_{\vartheta_{0}}}\right.$ : $\left.0 \leq t \leq \frac{1}{\varepsilon} T\right\}$.

Proof. Estimation of the global error (3.9) by means of the stability results given in Section 3.1 leads to

$$
\begin{aligned}
\left\|v_{N}-\widehat{u}_{N}\right\|_{X} \leq & \left\|u_{0}-\widehat{u}_{0}\right\|_{X}+\sum_{n=1}^{N}\left\|\mathcal{C}_{A+\varepsilon B}\left(N_{0} T_{0}\right) \widehat{u}_{n-1}-\mathcal{E}_{A+\varepsilon B}\left(N_{0} T_{0}\right) \widehat{u}_{n-1}\right\|_{X} \\
& +\sum_{n=1}^{N}\left\|\mathcal{D}_{A+\varepsilon B}\left(N_{0} T_{0}\right) u_{n-1}-\mathcal{C}_{A+\varepsilon B}\left(N_{0} T_{0}\right) u_{n-1}\right\|_{X} .
\end{aligned}
$$

The bound for the defect of a multi-revolution composition method, stated in Theorem 3.2, together with the relation $T=N H T_{0}$ at once implies

$$
\sum_{n=1}^{N}\left\|\mathcal{C}_{A+\varepsilon B}\left(N_{0} T_{0}\right) \widehat{u}_{n-1}-\mathcal{E}_{A+\varepsilon B}\left(N_{0} T_{0}\right) \widehat{u}_{n-1}\right\|_{X} \leq \frac{C}{(P+1) !} C_{B, 0}^{P+1} T T_{0}^{P} H^{P} \max \left\{\left\|\widehat{u}_{n}\right\|_{X}: 0 \leq n \leq N-1\right\} .
$$

Theorem 3.4 yields the error estimate

$$
\sum_{n=1}^{N}\left\|\mathcal{D}_{A+\varepsilon B}\left(N_{0} T_{0}\right) u_{n-1}-\mathcal{C}_{A+\varepsilon B}\left(N_{0} T_{0}\right) u_{n-1}\right\|_{X} \leq C\left(h^{m}+H h^{p}\right) \max \left\{\left\|u_{n}\right\|_{X_{\max \{m, p\} / 2}}: 0 \leq n \leq N-1\right\}
$$

and the improved result for symmetric splitting methods

$$
\sum_{n=1}^{N}\left\|\mathcal{D}_{A+\varepsilon B}\left(N_{0} T_{0}\right) u_{n-1}-\mathcal{C}_{A+\varepsilon B}\left(N_{0} T_{0}\right) u_{n-1}\right\|_{X} \leq C\left(h^{m}+\varepsilon h^{p}\right) \max \left\{\left\|u_{n}\right\|_{X_{\max \{m, p\} / 2}}: 0 \leq n \leq N-1\right\} .
$$

Altogether, this proves the stated convergence estimate. 


\section{NumERICAL EXPERIMENTS}

In this section, we illustrate the convergence behaviour of multi-revolution composition methods combined with time-splitting methods for linear and nonlinear Schrödinger equations. In order to confirm the dependence of our global error estimate with respect to the increment $H$ and time stepsize $h$, we first consider problems in a single space dimension, which facilitates the numerical tests; as a further illustration, we include the results for a two-dimensional problem. The space discretisation based on the Fourier pseudo-spectral method is chosen such that the spatial error is negligible. Further numerical experiments illustrating the favourable behaviour of time-splitting pseudo-spectral methods for the space discretisation of this type of problems are found in $[8,19]$, see also references given therein.

\subsection{Linear and nonlinear test equations (1D)}

We consider a linear Schrödinger equation imposing a periodic bounded real-valued potential and a periodic initial state

$$
\begin{gathered}
\mathrm{i} \partial_{t} \psi(x, t)=-\partial_{x x} \psi(x, t)+\varepsilon V(x) \psi(x, t), \\
V(x)=2 \cos (2 x), \quad \psi(x, 0)=\sin x+\cos x, \quad(x, t) \in(0,2 \pi) \times\left(0, \frac{1}{\varepsilon} T\right] .
\end{gathered}
$$

In the present situation, the requirements of Hypothesis 2.1 are satisfied with $T_{0}=2 \pi$; moreover, it is ensured that the exact solution remains bounded in higher fractional power spaces which correspond to Sobole $v$-spaces of higher degree. In addition, we consider the nonlinear test equation

$$
\mathrm{i} \partial_{t} \psi(x, t)=-\partial_{x x} \psi(x, t)+\varepsilon V(x)|\psi(x, t)|^{2} \psi(x, t), \quad(x, t) \in(0,2 \pi) \times\left(0, \frac{1}{\varepsilon} T\right],
$$

with function $V$ and initial state chosen as above.

\subsection{Space and time discretisation}

For the spatial discretisation of (4.1)-(4.2) we apply the Fourier pseudo-spectral method with $M=256$ basis function; hence, the spatial error can be considered as insignificant. The time discretisation (2.8) relies on the second-order multi-revolution composition method (1.4) and the fourth-order method (1.3); for their realisation, we apply the widely used symmetric second-order Strang splitting method (1.6), a non-symmetric second-order splitting method with coefficients

$$
p=2: \quad s=2, \quad a_{1}=\frac{1}{6}, \quad a_{2}=1-a_{1}, \quad b_{1}=\frac{3}{5}, \quad b_{2}=1-b_{1},
$$

and a symmetric fourth-order splitting method constructed by Yoshida [22]. We recall that $H=\varepsilon N_{0}$ with integer $N, N_{0}>0$ such that $T=N H T_{0}$; provided that the time stepsizes is chosen in the form $h=T_{0} / K$ for integer $K>0$, an improved error behaviour can be expected for symmetric splitting methods. Reference solutions $\psi^{\mathrm{ref}}(T)$ at the final time $T=\frac{\pi}{4}$ are computed by means of the fourth-order splitting method, applied with time stepsize $\Delta t=2 \pi \times 10^{-4}$. The approximation errors are measured with respect to the discrete $\ell^{2}$-norm.

\subsection{Numerical results}

In Figure 3, the global errors of the second-order and fourth-order multi-revolution composition methods combined with the fourth-order splitting method, obtained for the linear and nonlinear test equations, are displayed; for clarity, the numerical results are also given in Table 1. For the fixed time stepsize $h=T_{0} \times 10^{-3}$ 

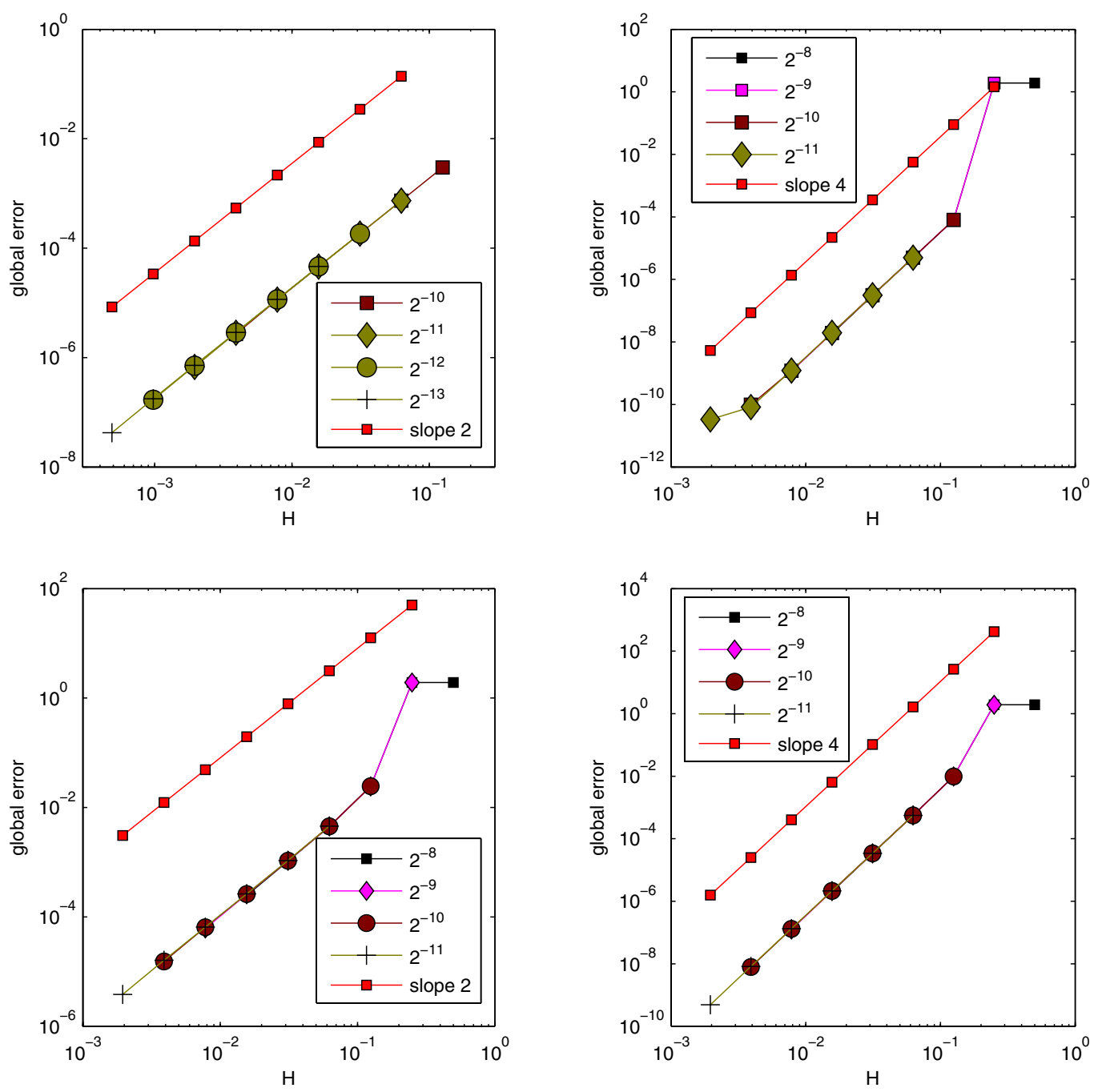

Figure 3. Time integration of linear (first row) and nonlinear (second row) test equations (1D) by a second-order (left) and a fourth-order (right) MRCM, respectively, combined with a fourth-order splitting method applied with stepsize $h=T_{0} \times 10^{-3}$. Global errors with respect to numerical reference solutions versus increments $H$ for $\varepsilon=2^{-10-j}$ with $j \in\{0,1,2,3\}$ or $\varepsilon=2^{-8-j}$ with $j \in\{0,1,2,3\}$, respectively. Reference lines of slopes 2 and 4 are drawn correspondingly. See Table 1.

the error caused by the splitting method is relatively small compared to the error caused by the multi-revolution composition methods. In Figure 4, the global errors of the second-order multi-revolution method combined with the non-symmetric versus the symmetric second-order splitting method are displayed; the corresponding results are also given in Table 2. In Figure 5, the global errors of the fourth-order multi-revolution method combined with the symmetric fourth-order splitting method are given. All numerical results are in accordance with our convergence estimate; indeed in the non-symmetric case, we expect the global error to be dominated by the terms $\mathcal{O}\left(H^{P}\right)+\mathcal{O}\left(H h^{p}\right)$, and in the symmetric case, we expect the global error behaviour $\mathcal{O}\left(H^{P}\right)+\mathcal{O}\left(\varepsilon h^{p}\right)$. 

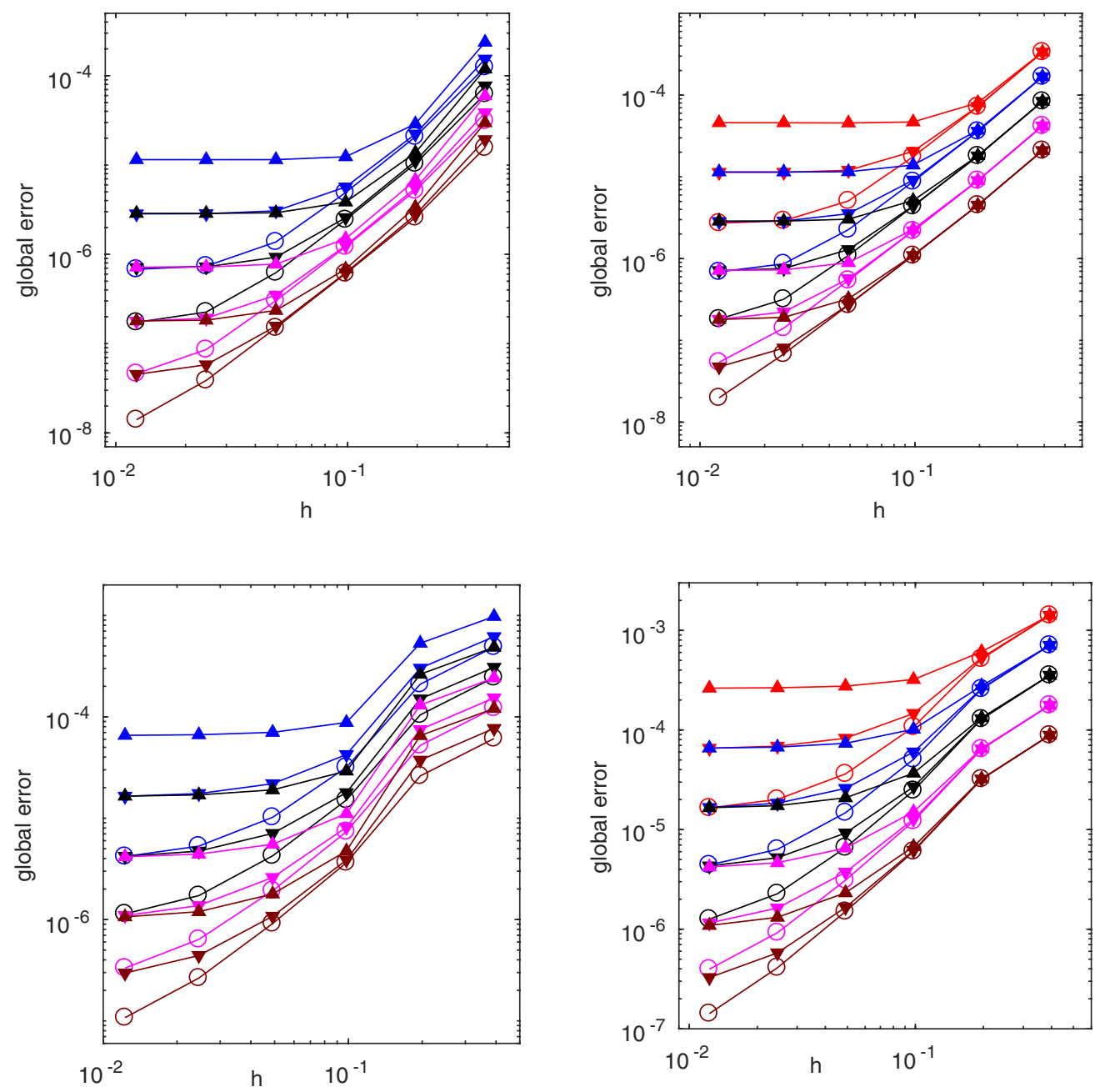

Figure 4. Time integration of linear (first row) and nonlinear (second row) test equations (1D) by a second-order MRCM combined with a non-symmetric or a symmetric second-order splitting method, respectively. Global errors with respect to numerical reference solutions versus time stepsizes $h$. Non-symmetric case (left): Lines of same color represent errors for same $\varepsilon$ but different increments $H=8 \varepsilon$ (circle), $16 \varepsilon$ (downward-pointing triangle), $32 \varepsilon$ (upward-pointing triangle) for $\varepsilon=2^{-11-j}$ with $j \in\{0,1,2,3\}$. Symmetric case (right): Lines of same color represent errors for same $\varepsilon$ but different increments $H=4 \varepsilon$ (circle), $8 \varepsilon$ (downward-pointing triangle), $16 \varepsilon$ (upward-pointing triangle) for $\varepsilon=2^{-10-j}$ with $j \in\{0,1,2,3,4\}$. See Table 2.

\subsection{Linear test equation (2D)}

As an extension of (4.1), we consider the two-dimensional linear test equation

$$
\begin{gathered}
\text { i } \partial_{t} \psi(x, y, t)=-\Delta \psi(x, y, t)+\varepsilon V(x, y) \psi(x, y, t), \\
V(x, y)=2 \cos (2 x) \sin (4 y), \quad \psi(x, 0)=(\sin x+\cos x) \cos (y), \\
(x, y, t) \in(0,2 \pi) \times(0,2 \pi) \times\left[0, \frac{1}{\varepsilon} T\right] .
\end{gathered}
$$



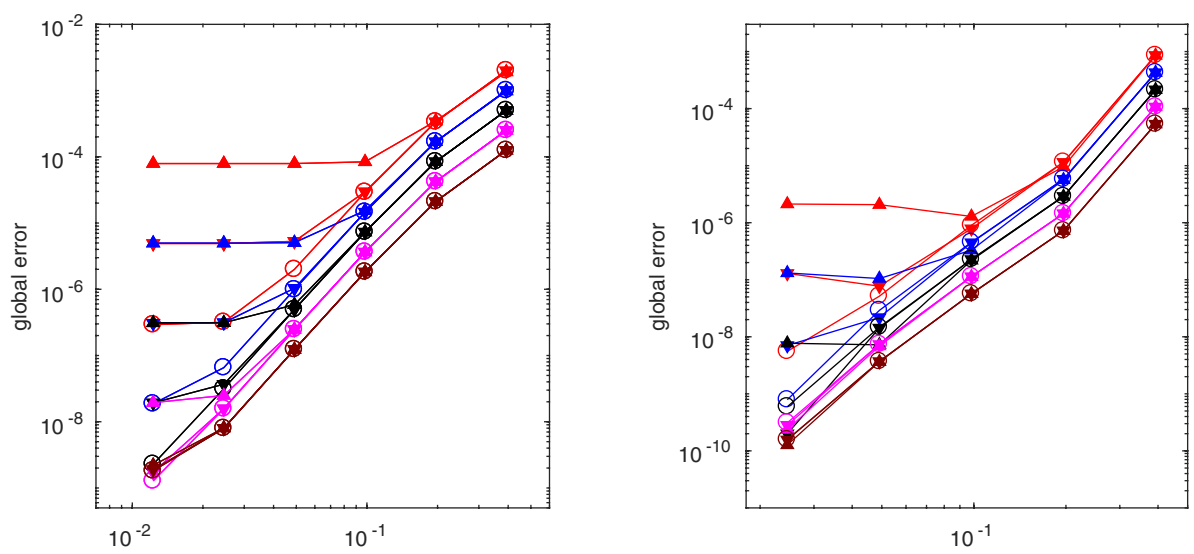

FIGURE 5. Time integration of linear (left) and nonlinear (right) test equations (1D) by a fourthorder MRCM combined with a symmetric fourth-order splitting method. Global errors with respect to numerical reference solutions versus time stepsizes $h$. Lines of same color represent errors for same $\varepsilon$ but different increments $H=4 \varepsilon$ (circle), $8 \varepsilon$ (downward-pointing triangle, $16 \varepsilon$ (upward-pointing triangle) for $\varepsilon=2^{-10-j}$ with $j \in\{0,1,2,3,4\}$.
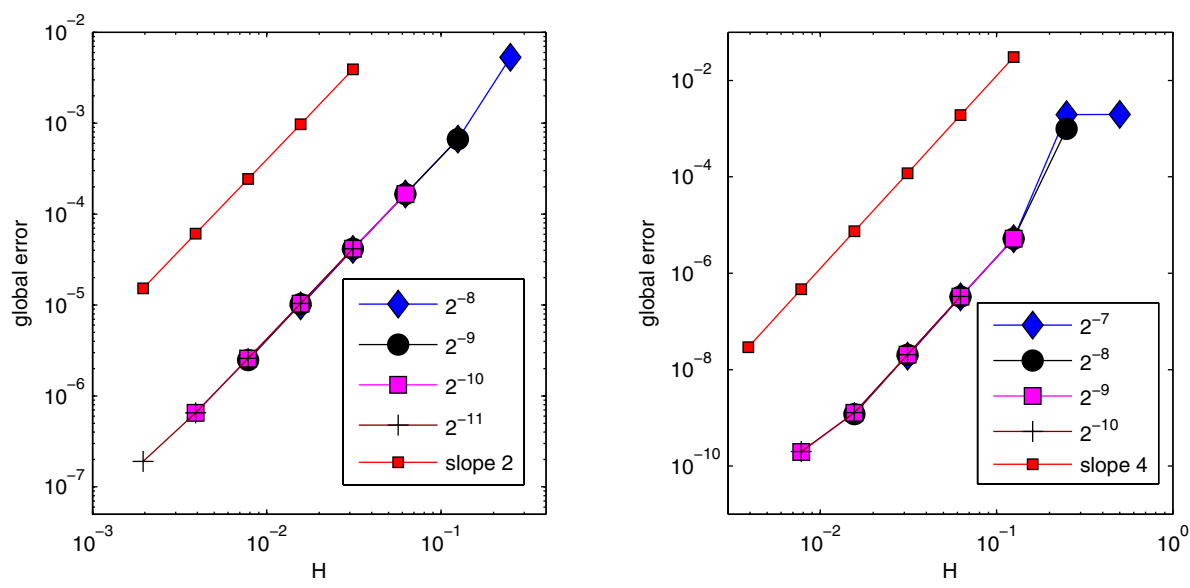

FIgURE 6. Time integration of a linear test equation (2D) by a second-order MRCM combined with the second-order Strang splitting method (left) and a fourth-order MRCM combined with the fourth-order splitting method by Yoshida (right), respectively, applied with stepsize $h=$ $T_{0} \cdot 10^{-3}$. Global errors with respect to numerical reference solutions versus increments $H$ for $\varepsilon=2^{-8-j}$ with $j \in\{0,1,2,3\}$ or $\varepsilon=2^{-7-j}$ with $j \in\{0,1,2,3\}$, respectively. Reference lines of slopes 2 and 4 are drawn correspondingly.

For the spatial discretisation, we apply the Fourier pseudo-spectral method with $M_{x}=M_{y}=256$ basis function; the time discretisation relies on the second-order multi-revolution composition method combined with the symmetric second-order Strang splitting method and the fourth-order multi-revolution composition method combined with the symmetric fourth-order Yoshida splitting method. Reference solutions $\psi^{\text {ref }}(T)$ at time $T=\frac{\pi}{4}$ are computed by the fourth-order splitting method, applied with time stepsize $\Delta t=2 \pi \times 10^{-3}$; the approximation errors are measured with respect to the discrete $\ell^{2}$-norm. The numerical results are displayed in Figures 6 and 7 ; as expected, they confirm our convergence estimate and qualitatively conform to the one-dimensional case. 

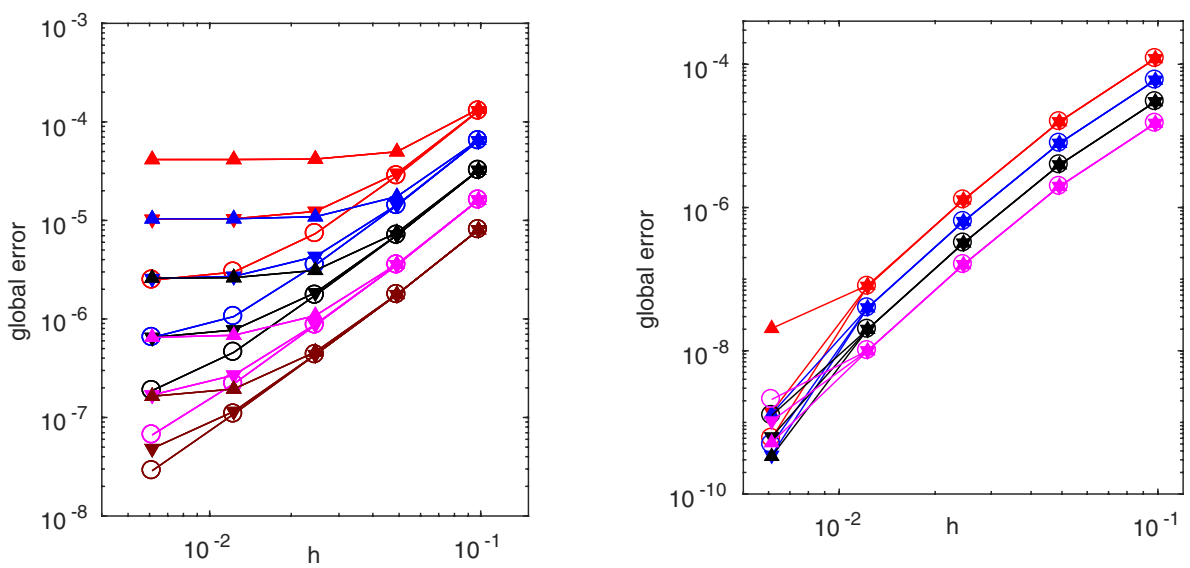

FiguRE 7. Time integration of a linear test equation (2D) by a second-order MRCM combined with the second-order Strang splitting method (left) and a fourth-order MRCM combined with the fourth-order splitting method by Yoshida (right), respectively. Global errors with respect to numerical reference solutions versus time stepsizes $h$. Lines of same color represent errors for same $\varepsilon$ but different increments $H=4 \varepsilon$ (circle), $8 \varepsilon$ (downward-pointing triangle), $16 \varepsilon$ (upward-pointing triangle) for $\varepsilon=2^{-9-j}$ with $j \in\{0,1,2,3,4\}$ (left) and $H=8 \varepsilon$ (circle), $16 \varepsilon$ (downward-pointing triangle), $32 \varepsilon$ (upward-pointing triangle) for $\varepsilon=2^{-9-j}$ with $j \in\{0,1,2,3\}$ (right).

TABLE 1. Time integration of linear and nonlinear test equations (1D) by a second-order $(P=2)$ and a fourth-order $(P=4)$ MRCM, respectively, combined with a fourth-order splitting method applied with stepsize $h=T_{0} \times 10^{-3}$. Global errors with respect to numerical reference solutions in dependence of the increments $H=\varepsilon N_{0}$. See Figure 3 .

\begin{tabular}{ccccccc}
\hline \multicolumn{7}{c}{ Linear test equation } \\
\hline$P=2$ & $N_{0}=2^{2}$ & $N_{0}=2^{3}$ & $N_{0}=2^{4}$ & $N_{0}=2^{5}$ & $N_{0}=2^{6}$ & $N_{0}=2^{7}$ \\
\hline$\varepsilon=2^{-10}$ & $2.704 \mathrm{e}-6$ & $1.136 \mathrm{e}-5$ & $4.598 \mathrm{e}-5$ & $1.846 \mathrm{e}-4$ & $7.405 \mathrm{e}-4$ & $2.991 \mathrm{e}-3$ \\
$\varepsilon=2^{-11}$ & $6.761 \mathrm{e}-7$ & $2.839 \mathrm{e}-6$ & $1.149 \mathrm{e}-5$ & $4.611 \mathrm{e}-5$ & $1.847 \mathrm{e}-4$ & $7.407 \mathrm{e}-4$ \\
$\varepsilon=2^{-12}$ & $1.691 \mathrm{e}-7$ & $7.099 \mathrm{e}-7$ & $2.873 \mathrm{e}-6$ & $1.153 \mathrm{e}-5$ & $4.615 \mathrm{e}-5$ & $1.847 \mathrm{e}-4$ \\
$\varepsilon=2^{-13}$ & $4.225 \mathrm{e}-8$ & $1.775 \mathrm{e}-7$ & $7.183 \mathrm{e}-7$ & $2.882 \mathrm{e}-6$ & $1.154 \mathrm{e}-5$ & $4.616 \mathrm{e}-5$ \\
\hline$P=4$ & $N_{0}=2^{2}$ & $N_{0}=2^{3}$ & $N_{0}=2^{4}$ & $N_{0}=2^{5}$ & $N_{0}=2^{6}$ & $N_{0}=2^{7}$ \\
\hline$\varepsilon=2^{-8}$ & $1.824 \mathrm{e}-8$ & $3.071 \mathrm{e}-7$ & $4.968 \mathrm{e}-6$ & $7.936 \mathrm{e}-5$ & 1.919 & 1.919 \\
$\varepsilon=2^{-9}$ & $1.148 \mathrm{e}-9$ & $1.920 \mathrm{e}-8$ & $3.109 \mathrm{e}-7$ & $4.984 \mathrm{e}-6$ & $7.942 \mathrm{e}-5$ & 1.919 \\
$\varepsilon=2^{-10}$ & $9.822 \mathrm{e}-11$ & $1.202 \mathrm{e}-9$ & $1.944 \mathrm{e}-8$ & $3.119 \mathrm{e}-7$ & $4.987 \mathrm{e}-6$ & $7.943 \mathrm{e}-5$ \\
$\varepsilon=2^{-11}$ & $3.349 \mathrm{e}-11$ & $8.206 \mathrm{e}-11$ & $1.216 \mathrm{e}-9$ & $1.950 \mathrm{e}-8$ & $3.121 \mathrm{e}-7$ & $4.988 \mathrm{e}-6$ \\
\hline & & \multicolumn{5}{c}{} \\
\hline$P=2$ & $N_{0}=2^{2}$ & $N_{0}=2^{3}$ & $N_{0}=2^{4}$ & $N_{0}=2^{5}$ & $N_{0}=2^{6}$ & $N_{0}=2^{7}$ \\
\hline$\varepsilon=2^{-8}$ & $2.455 \mathrm{e}-4$ & $1.046 \mathrm{e}-3$ & $4.495 \mathrm{e}-3$ & $2.426 \mathrm{e}-2$ & 1.923 & 1.919 \\
$\varepsilon=2^{-9}$ & $6.136 \mathrm{e}-5$ & $2.586 \mathrm{e}-4$ & $1.062 \mathrm{e}-3$ & $4.524 \mathrm{e}-3$ & $2.436 \mathrm{e}-2$ & 1.923 \\
$\varepsilon=2^{-10}$ & $1.535 \mathrm{e}-5$ & $6.453 \mathrm{e}-5$ & $2.621 \mathrm{e}-4$ & $1.067 \mathrm{e}-3$ & $4.534 \mathrm{e}-3$ & $2.441 \mathrm{e}-2$ \\
$\varepsilon=2^{-11}$ & $3.840 \mathrm{e}-6$ & $1.613 \mathrm{e}-5$ & $6.534 \mathrm{e}-5$ & $2.631 \mathrm{e}-4$ & $1.068 \mathrm{e}-3$ & $4.539 \mathrm{e}-3$ \\
\hline$P=4$ & $N_{0}=2^{2}$ & $N_{0}=2^{3}$ & $N_{0}=2^{4}$ & $N_{0}=2^{5}$ & $N_{0}=2^{6}$ & $N_{0}=2^{7}$ \\
\hline$\varepsilon=2^{-8}$ & $2.022 \mathrm{e}-6$ & $3.386 \mathrm{e}-5$ & $5.556 \mathrm{e}-4$ & $9.809 \mathrm{e}-3$ & 1.920 & 1.919 \\
$\varepsilon=2^{-9}$ & $1.262 \mathrm{e}-7$ & $2.107 \mathrm{e}-6$ & $3.416 \mathrm{e}-5$ & $5.564 \mathrm{e}-4$ & $9.801 \mathrm{e}-3$ & 1.921 \\
$\varepsilon=2^{-10}$ & $7.879 \mathrm{e}-9$ & $1.315 \mathrm{e}-7$ & $2.127 \mathrm{e}-6$ & $3.423 \mathrm{e}-5$ & $5.564 \mathrm{e}-4$ & $9.797 \mathrm{e}-3$ \\
$\varepsilon=2^{-11}$ & $4.920 \mathrm{e}-10$ & $8.215 \mathrm{e}-9$ & $1.328 \mathrm{e}-7$ & $2.132 \mathrm{e}-6$ & $3.424 \mathrm{e}-5$ & $5.564 \mathrm{e}-4$ \\
\hline
\end{tabular}


TABLE 2. Time integration of linear and nonlinear test equations (1D) by a second-order MRCM combined with a non-symmetric versus a second-order splitting method. Global errors with respect to numerical reference solutions. See Figure 4.

\begin{tabular}{|c|c|c|c|c|c|c|}
\hline \multicolumn{7}{|c|}{ Linear test equation } \\
\hline Non-sym & etric case & $h=T_{0} / 2^{4}$ & $h=T_{0} / 2^{5}$ & $h=T_{0} / 2^{6}$ & $h=T_{0} / 2^{7}$ & $h=T_{0} / 2^{8}$ \\
\hline \multirow{3}{*}{$\varepsilon=2^{-12}$} & $N_{0}=32$ & $2.1674 \mathrm{e}-4$ & $2.2936 \mathrm{e}-5$ & $1.1679 \mathrm{e}-5$ & $1.1471 \mathrm{e}-5$ & $1.1509 \mathrm{e}-5$ \\
\hline & $N_{0}=16$ & $1.1878 \mathrm{e}-4$ & $1.3719 \mathrm{e}-5$ & $3.8496 \mathrm{e}-6$ & $2.9196 \mathrm{e}-6$ & $2.8724 \mathrm{e}-6$ \\
\hline & $N_{0}=8$ & $7.6928 \mathrm{e}-5$ & $1.1060 \mathrm{e}-5$ & $2.5726 \mathrm{e}-6$ & $9.2760 \mathrm{e}-7$ & $7.2437 \mathrm{e}-7$ \\
\hline \multirow{3}{*}{$\varepsilon=2^{-13}$} & $N_{0}=32$ & $1.0841 \mathrm{e}-4$ & $1.0572 \mathrm{e}-5$ & $3.2363 \mathrm{e}-6$ & $2.8815 \mathrm{e}-6$ & $2.8779 \mathrm{e}-6$ \\
\hline & $N_{0}=16$ & $5.9452 \mathrm{e}-5$ & $6.7697 \mathrm{e}-6$ & $1.4957 \mathrm{e}-6$ & $7.7663 \mathrm{e}-7$ & $7.2102 \mathrm{e}-7$ \\
\hline & $N_{0}=8$ & $3.8492 \mathrm{e}-5$ & $5.5233 \mathrm{e}-6$ & $1.2509 \mathrm{e}-6$ & $3.4896 \mathrm{e}-7$ & $1.9227 \mathrm{e}-7$ \\
\hline \multirow{3}{*}{$\varepsilon=2^{-14}$} & $N_{0}=32$ & $5.4234 \mathrm{e}-5$ & $5.1676 \mathrm{e}-6$ & $1.0687 \mathrm{e}-6$ & $7.3413 \mathrm{e}-7$ & $7.2022 \mathrm{e}-7$ \\
\hline & $N_{0}=16$ & $2.9744 \mathrm{e}-5$ & $3.3734 \mathrm{e}-6$ & $6.8373 \mathrm{e}-7$ & $2.3514 \mathrm{e}-7$ & $1.8314 \mathrm{e}-7$ \\
\hline & $N_{0}=8$ & $1.9253 \mathrm{e}-5$ & $2.7607 \mathrm{e}-6$ & $6.2090 \mathrm{e}-7$ & $1.5683 \mathrm{e}-7$ & $5.7897 \mathrm{e}-8$ \\
\hline \multicolumn{2}{|c|}{ Symmetric case } & $h=T_{0} / 2^{4}$ & $h=T_{0} / 2^{5}$ & $h=T_{0} / 2^{6}$ & $h=T_{0} / 2^{7}$ & $h=T_{0} / 2^{8}$ \\
\hline \multirow{3}{*}{$\varepsilon=2^{-11}$} & $N_{0}=16$ & $1.6779 \mathrm{e}-4$ & $3.7116 \mathrm{e}-5$ & $1.3948 \mathrm{e}-5$ & $1.1548 \mathrm{e}-5$ & $1.1468 \mathrm{e}-5$ \\
\hline & $N_{0}=8$ & $1.6778 \mathrm{e}-4$ & $3.6074 \mathrm{e}-5$ & $9.1198 \mathrm{e}-6$ & $3.5389 \mathrm{e}-6$ & $2.8809 \mathrm{e}-6$ \\
\hline & $N_{0}=4$ & $1.6778 \mathrm{e}-4$ & $3.6009 \mathrm{e}-5$ & $8.7379 \mathrm{e}-6$ & $2.2622 \mathrm{e}-6$ & $8.6306 \mathrm{e}-7$ \\
\hline \multirow{3}{*}{$\varepsilon=2^{-12}$} & $N_{0}=16$ & $8.3891 \mathrm{e}-5$ & $1.8143 \mathrm{e}-5$ & $5.1381 \mathrm{e}-6$ & $3.0349 \mathrm{e}-6$ & $2.8765 \mathrm{e}-6$ \\
\hline & $N_{0}=8$ & $8.3890 \mathrm{e}-5$ & $1.8012 \mathrm{e}-5$ & $4.4090 \mathrm{e}-6$ & $1.2882 \mathrm{e}-6$ & $7.5727 \mathrm{e}-7$ \\
\hline & $N_{0}=4$ & $8.3890 \mathrm{e}-5$ & $1.8003 \mathrm{e}-5$ & $4.3604 \mathrm{e}-6$ & $1.0938 \mathrm{e}-6$ & $3.1817 \mathrm{e}-7$ \\
\hline \multirow{3}{*}{$\varepsilon=2^{-13}$} & $N_{0}=16$ & $4.1945 \mathrm{e}-5$ & $9.0190 \mathrm{e}-6$ & $2.2824 \mathrm{e}-6$ & $8.9140 \mathrm{e}-7$ & $7.2852 \mathrm{e}-7$ \\
\hline & $N_{0}=8$ & $4.1945 \mathrm{e}-5$ & $9.0024 \mathrm{e}-6$ & $2.1851 \mathrm{e}-6$ & $5.6805 \mathrm{e}-7$ & $2.2238 \mathrm{e}-7$ \\
\hline & $N_{0}=4$ & $4.1945 \mathrm{e}-5$ & $9.0014 \mathrm{e}-6$ & $2.1790 \mathrm{e}-6$ & $5.4199 \mathrm{e}-7$ & $1.4123 \mathrm{e}-7$ \\
\hline \multicolumn{7}{|c|}{ Nonlinear test equation } \\
\hline \multicolumn{2}{|c|}{ Non-symmetric case } & $h=T_{0} / 2^{4}$ & $h=T_{0} / 2^{5}$ & $h=T_{0} / 2^{6}$ & $h=T_{0} / 2^{7}$ & $h=T_{0} / 2^{8}$ \\
\hline \multirow{3}{*}{$\varepsilon=2^{-12}$} & $N_{0}=32$ & $8.9539 \mathrm{e}-4$ & $5.0786 \mathrm{e}-4$ & $7.5686 \mathrm{e}-5$ & $6.7614 \mathrm{e}-5$ & $6.6061 \mathrm{e}-5$ \\
\hline & $N_{0}=16$ & $4.8553 \mathrm{e}-4$ & $2.6290 \mathrm{e}-4$ & $2.9169 \mathrm{e}-5$ & $1.9026 \mathrm{e}-5$ & $1.6974 \mathrm{e}-5$ \\
\hline & $N_{0}=8$ & $3.0798 \mathrm{e}-4$ & $1.5007 \mathrm{e}-4$ & $1.7851 \mathrm{e}-5$ & $7.0669 \mathrm{e}-6$ & $4.7344 \mathrm{e}-6$ \\
\hline \multirow{3}{*}{$\varepsilon=2^{-13}$} & $N_{0}=32$ & $4.4565 \mathrm{e}-4$ & $2.5170 \mathrm{e}-4$ & $2.2886 \mathrm{e}-5$ & $1.7617 \mathrm{e}-5$ & $1.6677 \mathrm{e}-5$ \\
\hline & $N_{0}=16$ & $2.4208 \mathrm{e}-4$ & $1.3081 \mathrm{e}-4$ & $1.1104 \mathrm{e}-5$ & $5.5264 \mathrm{e}-6$ & $4.4203 \mathrm{e}-6$ \\
\hline & $N_{0}=8$ & $1.5374 \mathrm{e}-4$ & $7.4814 \mathrm{e}-5$ & $8.1245 \mathrm{e}-6$ & $2.6001 \mathrm{e}-6$ & $1.3724 \mathrm{e}-6$ \\
\hline \multirow{3}{*}{$\varepsilon=2^{-14}$} & $N_{0}=32$ & $2.2242 \mathrm{e}-4$ & $1.2542 \mathrm{e}-4$ & $7.9512 \mathrm{e}-6$ & $4.7816 \mathrm{e}-6$ & $4.2559 \mathrm{e}-6$ \\
\hline & $N_{0}=16$ & $1.2088 \mathrm{e}-4$ & $6.5262 \mathrm{e}-5$ & $4.7412 \mathrm{e}-6$ & $1.7871 \mathrm{e}-6$ & $1.1959 \mathrm{e}-6$ \\
\hline & $N_{0}=8$ & $7.6811 \mathrm{e}-5$ & $3.7353 \mathrm{e}-5$ & $3.8688 \mathrm{e}-6$ & $1.0773 \mathrm{e}-6$ & $4.4191 \mathrm{e}-7$ \\
\hline \multicolumn{2}{|c|}{ Symmetric case } & $h=T_{0} / 2^{4}$ & $h=T_{0} / 2^{5}$ & $h=T_{0} / 2^{6}$ & $h=T_{0} / 2^{7}$ & $h=T_{0} / 2^{8}$ \\
\hline \multirow{3}{*}{$\varepsilon=2^{-11}$} & $N_{0}=16$ & $7.0791 \mathrm{e}-4$ & $2.7695 \mathrm{e}-4$ & $1.0184 \mathrm{e}-4$ & $7.3457 \mathrm{e}-5$ & $6.7292 \mathrm{e}-5$ \\
\hline & $N_{0}=8$ & $7.0789 \mathrm{e}-4$ & $2.6092 \mathrm{e}-4$ & $6.0151 \mathrm{e}-5$ & $2.5910 \mathrm{e}-5$ & $1.8384 \mathrm{e}-5$ \\
\hline & $N_{0}=4$ & $7.0789 \mathrm{e}-4$ & $2.5774 \mathrm{e}-4$ & $5.0710 \mathrm{e}-5$ & $1.4767 \mathrm{e}-5$ & $6.3339 \mathrm{e}-6$ \\
\hline \multirow{3}{*}{$\varepsilon=2^{-12}$} & $N_{0}=16$ & $3.5395 \mathrm{e}-4$ & $1.3287 \mathrm{e}-4$ & $3.6842 \mathrm{e}-5$ & $2.0849 \mathrm{e}-5$ & $1.7393 \mathrm{e}-5$ \\
\hline & $N_{0}=8$ & $3.5394 \mathrm{e}-4$ & $1.2940 \mathrm{e}-4$ & $2.6939 \mathrm{e}-5$ & $9.2361 \mathrm{e}-6$ & $5.2156 \mathrm{e}-6$ \\
\hline & $N_{0}=4$ & $3.5394 \mathrm{e}-4$ & $1.2864 \mathrm{e}-4$ & $2.4654 \mathrm{e}-5$ & $6.5860 \mathrm{e}-6$ & $2.2703 \mathrm{e}-6$ \\
\hline \multirow{3}{*}{$\varepsilon=2^{-13}$} & $N_{0}=16$ & $1.7697 \mathrm{e}-4$ & $6.5243 \mathrm{e}-5$ & $1.5078 \mathrm{e}-5$ & $6.5247 \mathrm{e}-6$ & $4.6452 \mathrm{e}-6$ \\
\hline & $N_{0}=8$ & $1.7697 \mathrm{e}-4$ & $6.4446 \mathrm{e}-5$ & $1.2714 \mathrm{e}-5$ & $3.7333 \mathrm{e}-6$ & $1.6299 \mathrm{e}-6$ \\
\hline & $N_{0}=4$ & $1.7697 \mathrm{e}-4$ & $6.4260 \mathrm{e}-5$ & $1.2153 \mathrm{e}-5$ & $3.1011 \mathrm{e}-6$ & $9.2182 \mathrm{e}-7$ \\
\hline
\end{tabular}


Acknowledgements. We acknowledge financial support by the Agence nationale de la recherche (ANR and FWF) within the Blanc International II Programme under project Modeling and Numerical Simulation of Low Dimensional Quantum Systems (LODIQUAS), by the Austrian Science Fund (FWF) under SFB Vienna Computational Materials Laboratory (ViCoM) and project P21620-N13, and by the Austrian Ministry of Science and Research via its grant for the WPI. The presented numerical results have been achieved by using the Vienna Scientific Cluster.

\section{REFERENCES}

[1] R.A. Adams, Sobolev Spaces. Academic Press, Orlando, Florida (1975).

[2] W. Bao, Mathematical models and numerical methods for Bose-Einstein condensation. In Vol. IV of Proc. Inter. Congress Math. Seoul (2014) 971-996.

[3] W. Bao, P. Markowich, Ch. Schmeiser and R.M. Weishäupl, On the Gross-Pitaevski equation with strongly anisotropic confinement: formal asymptotics and numerical experiments. M3AS 15 (2005) 767-782.

[4] N. Ben Abdallah, F. Méhats, Ch. Schmeiser and R.M. Weishäupl, The nonlinear Schrödinger equation with strong anisotropic harmonic potential. SIAM J. Math. Anal. 37 (2005) 189-199.

[5] S. Blanes and P.C. Moan, Practical symplectic partitioned Runge-Kutta and Runge-Kutta-Nyström methods. J. Comput. Appl. Math. 142 (2002) 313-330.

[6] R. Carles and E. Faou, Energy cascade for NLS on the torus. DCDS-A 32 (2012) 2063-2077.

[7] Ph. Chartier, J. Makazaga, A. Murua and G. Vilmart, Multi-revolution composition methods for highly oscillatory differential equations. Numer. Math. 128 (2014) 167-192.

[8] Ph. Chartier, F. Méhats, M. Thalhammer and Y. Zhang, Improved error estimates for splitting methods applied to highlyoscillatory nonlinear Schrödinger equations. Math. Comput. 85 (2016) 2863-2885.

[9] Ph. Chartier, N. Mauser, F. Méhats and Y. Zhang, Solving highly-oscillatory NLS with SAM: numerical efficiency and geometric properties. Discrete Contin. Dyn. Systems - Ser. S 9 (2016) 1327-1349.

[10] G. Dujardin and E. Faou, Normal form and long time analysis of splitting schemes for the linear Schrödinger equation with small potential. Numer. Math. 108 (2007) 223-262.

[11] K.-J. Engel and R. Nagel, One-Parameter Semigroups for Linear Evolution Equations. Springer, New York (2000).

[12] E. Faou, L. Gauckler and C. Lubich, Plane wave stability of the split-step Fourier method for the nonlinear Schrödinger equation. Forum Math. Sigma 2 (2014). Available at: doi:10.1017/fms.2014.4

[13] L. Gauckler, Convergence of a split-step Hermite method for the Gross-Pitaevskii equation. IMA J. Numer. Anal. 31 (2011) $396-415$.

[14] B. Grébert and L. Thomann, Resonant dynamics for the quintic nonlinear Schrödinger equation. Ann. Inst. Henri Poincaré Anal. Non Linéaire 29 (2012) 455-477.

[15] B. Grébert and C. Villegas-Blas, On the energy exchange between resonant modes in nonlinear Schrödinger equations. Ann. Inst. Henri Poincaré Anal. Non Linéaire 28 (2011) 127-134.

[16] H. Hofstätter, O. Koch and M. Thalhammer, Convergence analysis of high-order time-splitting pseudo-spectral methods for Gross-Pitaevskii equations with rotation term. Numer. Math. 127 (2014) 315-364.

[17] Ch. Lubich, On splitting methods for Schrödinger-Poisson and cubic nonlinear Schrödinger equations. Math. Comput. 77 (2008) 2141-2153.

[18] A. Pazy, Semigroups of Linear Operators and Applications to Partial Differential Equations. Appl. Math. Sci. Springer, New York 44 (1983).

[19] M. Thalhammer, Convergence analysis of high-order time-splitting pseudospectral methods for nonlinear Schrödinger equations. SIAM J. Numer. Anal. 50 (2013) 3231-3258.

[20] H. Triebel, Higher Analysis. Barth, Leipzig-Berlin-Heidelberg (1992).

[21] G. Vilmart, Weak second order multi-revolution composition methods for highly oscillatory stochastic differential equations with additive or multiplicative noise. SIAM J. Sci. Comput. 36 (2014) 1770-1796.

[22] H. Yoshida, Construction of higher order symplectic integrators. Phys. Lett. A 150 (1990) 262-268. 